OUTP-96-10S

ISAS/EP/96/23

$\mathrm{IC} / 96 / 26$

SWAT/95-96/98

\title{
Non-integrable Quantum Field Theories as Perturbations of Certain Integrable Models
}

\author{
G. Delfino ${ }^{a}$, G. Mussardo ${ }^{b, c, d}$ and P. Simonetti ${ }^{e}$ \\ a Theoretical Physics, University of Oxford \\ 1 Keble Road, Oxford OX1 3NP, UK \\ ${ }^{b}$ International School for Advanced Studies, \\ Via Beirut 3, 34014 Trieste, Italy \\ ${ }^{c}$ International Center of Theoretical Physics, \\ Strada Costiera 11, 34014 Trieste, Italy \\ d Istituto Nazionale di Fisica Nucleare, \\ Sezione di Trieste, Italy \\ e Department of Physics, University of Wales Swansea, \\ Singleton Park, Swansea SA2 8PP, UK
}

\begin{abstract}
We approach the study of non-integrable models of two-dimensional quantum field theory as perturbations of the integrable ones. By exploiting the knowledge of the exact $S$-matrix and Form Factors of the integrable field theories we obtain the first order corrections to the mass ratios, the vacuum energy density and the $S$-matrix of the non-integrable theories. As interesting applications of the formalism, we study the scaling region of the Ising model in an external magnetic field at $T \sim T_{c}$ and
\end{abstract}


the scaling region around the minimal model $M_{2,7}$. For these models, a remarkable agreement is observed between the theoretical predictions and the data extracted by a numerical diagonalization of their Hamiltonian. 


\section{Introduction}

Two-dimensional integrable and relativistic models have provided a most valuable heuristic guide in the analysis of the infinite-dimensional space of Quantum Field Theories and the associated statistical models. The peculiar property of the dynamics that makes those models solvable consists in the existence of an infinite number of integrals of motion. This feature manifests itself in several ways [1, 2]. First of all, it leads to very severe selection rules: for instance, scattering processes accompanied by production or annihilation events cannot take place in such theories and therefore the only allowed scattering processes are purely elastic. Secondly, the $n$-particle scattering amplitudes can always be factorized into the product of the $n(n-1) / 2$ two-particle elastic $S$-matrices. The twoparticle $S$-matrix $S_{a b}(s)$ for the particles $A_{a}$ and $A_{b}$, with mass $m_{a}$ and $m_{b}$ respectively, has only two elastic branch cut singularities at $s=\left(m_{a}-m_{b}\right)^{2}$ and $s=\left(m_{a}+m_{b}\right)^{2}$ and fulfills by itself the unitarity equation $S_{a b}(s) S_{a b}^{\dagger}(s)=1$ in the whole Riemannian surface of the Mandelstam variable $s$. Finally, the exact mass spectrum and the complete set of $S$-matrices of integrable QFT may be computed according to the so-called bootstrap principle which states that the bound states have to be regarded on the same footing as the asymptotic states. The drastic simplification of the on-shell properties of the integrable field theories has far-reaching consequences since it greatly facilitates both the computation of their thermodynamic quantities and the determination of their correlation functions (see, for instance [10-20]). For instance, the usual lengthy computation of matrix elements of local operators which enters the spectral representation of correlation functions can be shortened in the case of integrable models to solve a finite number of functional and recursive equations. At present, the exact $S$-matrix, the exact mass spectrum and the correlation functions of a large number of integrable models have been determined $\rrbracket^{\text {and }}$ confirmed either by analytic lattice computations or by numerical simulations [32-39]. One of the most remarkable examples of statistical model solved by using the bootstrap approach is represented by the Ising model in a magnetic field at $T=T_{c}$ : the exact $S$-matrix proposed by Zamolodchikov [2] has been the starting point

\footnotetext{
${ }^{1}$ For a brief list of references see $[1-20]$.
} 
for the solution of the long-standing problem of determining the spin-spin correlation function of this model [11].

Despite all the distinguishing qualities of integrable models and their successful applications to statistical mechanics, the actual realm of particle physics is however that of non-integrable QFT. These theories generally present the striking phenomenon of resonances as well as multi-production reactions. Many interesting statistical models in the vicinity of their fixed points may be described by non-integrable QFT and, to make any progress in the computation of their thermodynamical quantities (or, simply to understand better their qualitative features), it would be highly desirable to develop an appropriate formalism to deal with the lack of integrability. This task is notoriously difficult because the rich physical scenario of the multichannel systems, represented by the non-integrable field theories, is usually accompanied by great mathematical complexities. In fact, once one has given up the integrability condition, the infinite number of thresholds associated to the production processes greatly influences the analytic structure of the scattering amplitudes, inducing a rich pattern of branch cut singularities in addition to the pole structure generally associated to the bound or resonance states (Figure 1).

The mathematical difficulties of the problem are well known and cannot be easily circumvented, as illustrated by the following line of reasoning. Let $T_{1}<T_{2} \ldots<T_{n} \ldots$ be the infinite sequence of energy thresholds of the scattering amplitudes entering the $S$-matrix. In each interval $T_{n} \leq s<T_{n+1}$, the physical $S$-matrix is given by an $n \times n$ unitary matrix $S_{i j}^{(n)}(s)(i, j=1, \ldots, n)$, where here the suffixes $i$ and $j$ are multi-particle collective indices that generally label the different channels of the scattering process. At each threshold, a new channel opens up and correspondingly, more matrix elements have to be introduced. Due to the nested structure of the $S$-matrix as a function of $s$, i.e. $S^{(1)} \rightarrow S^{(2)} \ldots \rightarrow S^{(n)}$, one may be inclined to approach the difficult problem of computing the infinite number of scattering amplitudes by applying a recursive procedure. Namely, assuming that all the $n^{2}$ matrix elements $S_{i j}^{(n)}(s)$ have already been determined as analytic functions of $s$ in the interval $T_{n} \leq s<T_{n+1}$, one might try to analytically continue them into the next interval $T_{n+1} \leq s<T_{n+2}$ and then, as consequence, compute the new unknown scattering amplitudes entering the larger matrix $S_{i j}^{(n+1)}$ by using the 
unitarity equations relative to the new range of energies. There is, of course, an important flaw in the above argument: the recursive procedure is in fact based on the assumption that the matrix elements $S_{i j}^{(n)}(s)$ can be prolongated through the different thresholds without being halted by the presence of singularities, an assumption which is obviously false. Hence, the complexity of non-integrable QFT consists in the fact that in any energy interval one must take simultaneously into account all the inelastic thresholds of the theory. This feature is explicitly shown by a simple example discussed in Appendix A. For a discussion on the inter-related aspects of analyticity and integrability, see also ref. [27].

The problem of analysing non-integrable field theories (or at least a class thereof) is not however as impracticable as it may seem at first sight. A possible approach to their study is suggested by the observation that although integrable and non-integrable QFT possess quite different properties as far as scattering amplitudes and mass spectrum are concerned, their ultraviolet limit may be described by the same Conformal Field Theory (CFT). From this point of view, an integrable field theory is nothing more than a particular deformation of a Conformal Field Theory whose euclidean action may be written as

$$
\mathcal{A}_{\mathrm{int}}^{i}=\mathcal{A}_{\mathrm{CFT}}+g \int d^{2} x \Phi_{i}(x)
$$

where $\Phi_{i}(x)$ is one of the relevant operators which mantains the original integrability of the conformal model[2]. Starting now from a given conformal model, there are at least two ways of defining a non-integrable field theory: (a) the first possibility is to simply add a perturbation induced by a non-integrable operator to the CFT action ; (b) an alternative possibility is to deform the CFT action by means of a linear combination of several operators $\Phi_{i}$, in which at least one of these or all of them are individually

\footnotetext{
${ }^{2}$ Zamolodchikov has outlined some criteria on the integrability of the deformations of CFT. For a generic minimal model, for instance, integrable QFT are obtained as deformations by the relevant operators $\Phi_{1,3}, \Phi_{1,2}$ and $\Phi_{2,1}[2]$.
} 
integrable for the corresponding action in the last case we have

$$
\mathcal{A}=\mathcal{A}_{\mathrm{CFT}}+\sum_{j} g_{j} \int d^{2} x \Phi_{j}(x)
$$

Apart from numerical investigations [39] or perhaps standard analyses based on conformal perturbation methods, non-integrable QFT of type (a) are presently difficult to study and they will not be considered in this paper. On the contrary, non-integrable QFT of type (b) will be shown in this paper to be suitable of a purpose of theoretical analysis. This is particularly important because they provide in many respects the most interesting realization of non-integrable models. Let us illustrate the key observation that permits their analytic approach.

In order to study the non-integrable QFT associated to the euclidean action (1.2), it is convenient to regard the latter as the action relative to a deformation of an integrable QFT rather than a multiple deformation of CFT. This consists in grouping differently the terms in (1.2) and rewriting it as

$$
\mathcal{A}=\mathcal{A}_{\mathrm{int}}^{i}+\sum_{j \neq i} g_{j} \int d^{2} x \Phi_{j}(x)
$$

There are several advantages to adopting this point of view.

- Firstly, going to the Minkowski space, we can start our analysis of non-integrable QFT by using the particle basis of the integrable model associated to $\mathcal{A}_{\text {int }}^{i}$. Although their mass spectrum will not generally coincide, the particle basis of the latter model is surely more suitable than the conformal basis for capturing the large distance properties of the non-integrable theory团.

- Secondly, the integrable QFT with which we start our analysis are solvable theories, although not necessarly free. This means that we know how to compute the exact

\footnotetext{
${ }^{3}$ In the case when all the operators $\Phi_{i}$ are individually integrable but their simultaneous presence renders the field theory non-integrable, it can be argued that this is due to different null-vector structure of the fields $\Phi_{i}$ 四 or it can also be inferred by numerical studies of the spectrum, as discussed in the next sections.

${ }^{4}$ This is generally the case for massive field theories. For massless non-integrable field theories the analysis may be more subtle and will not be pursued here.
} 
matrix elements of all their local operators, in particular those entering the action (1.3). Hence, we can develop in this case a perturbative approach to non-integrable QFT based on the exact Form Factors of the integrable ones. As we will discuss in this paper, this approach seems generally more powerful and efficient than the one based on usual conformal perturbation theory. In fact, a large set of quantitative information -concerning the mass corrections, the renormalization of the vacuum energy density and the $S$-matrix of the non-integrable models- can already be obtained at the lowest order in the coupling constant $g_{j}$ with a high level of reliability引.

- Finally, in the case of multiple deformation of CFT by separately integrable operators, we can choose any of them for defining our initial solvable QFT. Obviously, each choice leads to a selection of particle basis, bound state structure and dynamics, on which we can construct our perturbative approach. However, since the actual dynamics of non-integrable QFT must be independent of the solvable model we start with, this implies that perturbative expansions based on different Form Factors and spectra must be related each other by a set of mathematical identities.

The most natural interpretation of eq. (1.2) when all $\Phi_{i}(x)$ are individually integrable operators, is then that of an interpolating action between exactly solvable models, the interpolation being realized by varying the relative values of the coupling constants $g_{i}$ (Figure 2). A remarkable example of such interpolating theory is provided by the Ising model away from the critical point, i.e. at $T \neq T_{c}$ and in a presence of a magnetic field $h$. In the pure magnetic direction, the spectrum of this model consists of eight stable particles, five of which above threshold [2]. In the pure temperature direction, on the contrary, the spectrum presents only one massive state, which can be interpreted either as a kink (in the low-temperature phase) or as a particle (in the high-temperature phase) [22-26]. The interpolation between the Hilbert spaces constructed on these rather different bases and the rich physical scenario associated to it will be analysed in Section 5.

${ }^{5}$ The formulas which we will derive in Section 3 can be regarded as "Born approximation formulas" for a non-integrable QFT. 
At this stage of the discussion, the careful reader may have noticed that we could have directly introduced the non-integrable QFT by means of the action (1.3) without further specifying how the integrable action $\mathcal{A}_{\text {int }}^{i}$ has been actually obtained. This is indeed true and in fact, all the formalism set up in this paper to study non-integrable models basically relies on the properties of relativistic integrable models as defined, for instance, by the framework of the analytic theory of the $S$-matrix. Although the perturbed CFT approach we have used in this section may then appear only as a convenient way to introduce and define non-integrable QFT, however the eventual knowledge of the underlying conformal model actually adds an important piece of information on the operator content and the ultraviolet properties of the theory. In fact, by the knowledge of the CFT ruling the short-distance Operator Product Expansion of the theory, one can severerly constrain its high-energy behaviour and obtain, among other things, a bound on the cross-sections of non-integrable QFT. This aspect as well as others of non-integrable models will be discussed in a separate publication [30].

The paper is organized as follows. In Section 2 we define the perturbative scheme based on the so-called intermediate state representation and the Form Factors of integrable theories. We also discuss the necessity of introducing some counterterms in the perturbative series in order to have a consistent formulation of the theory. In Section 3 we specify our analysis to the case of two-dimensional integrable QFT and derive the main formulas which rule the variation of the mass ratios, the vacuum energy density and the $S$-matrix. Section 4 is devoted to the simplest model which can be discussed by means of these techniques, namely the minimal model $\mathcal{M}_{2,7}$ perturbed by the operators $\Phi_{1,2}$ and $\Phi_{1,3}$. Another example of non-integrable quantum field theory is given by the Ising model away from criticality, at a generic point in the bidimensional phase diagram spanned by the magnetic field $h$ and the reduced temperature $\left(T-T_{c}\right) / T_{c}$. In view of its important role in statistical mechanics, the Ising model away from criticality will be discussed in some detail in Section 5. In particular, we will compute the first order corrections to the spectrum of the theory when a small thermal perturbation is added to the pure magnetic case. Our conclusions are then reported in Section 6. Three appendices complete the paper: the first one shows how the inelastic thresholds enter the elastic scattering 
amplitude of a simple example of quantum mechanics; the second appendix discusses the Faddeev-Zamolodchikov algebra and the disconnected terms present in the matrix elements of local operators; the last one gathers the relevant Form Factor expressions for the $\Phi_{1,3}$ deformation of the $M_{(2,7)}$ model.

\section{The Intermediate State Representation}

Let us consider a quantum field theory in the Minkowski space, defined by the action

$$
\mathcal{A}=\mathcal{A}_{0}+\mathcal{A}_{I}=\mathcal{A}_{0}-\lambda \int d^{2} x \Psi(x)
$$

where $\mathcal{A}_{0}$ denotes here the minkowskian action of the unperturbed theory and $\Psi$ one of its operators. We suppose that the QFT associated to the action $\mathcal{A}_{0}$ is exactly solvable (although not necessarily free), i.e. we assume that the spectrum of particles, their scattering amplitudes and the matrix elements of the operators of the theory (and in particular those of $\Psi$ ) are all known. For the sake of simplicity, we consider in this section the case of isospectral perturbations of a solvable theory. This means that the spectrum of the theory described by the total action $\mathcal{A}$ will be made of the same number of particles of the unperturbed one; in other words, the new interaction $\mathcal{A}_{I}$ only effects the values of the masses of the physical particles but not their stability properties ?

Let us now describe the properties of the theory associated to the action $\mathcal{A}$. Under the hypothesys that the interaction term is turned off at $t \rightarrow \pm \infty$, it is possible to adopt the formalism of the asymptotic "in" and "out" states. We are interested in computing the scattering amplitude

$$
S\left\{q_{1}, \ldots, q_{n} \rightarrow q_{1}^{\prime}, \ldots, q_{m}^{\prime}\right\}={ }^{\text {out }}\left\langle q_{1}^{\prime}, \ldots, q_{m}^{\prime} \mid q_{1}, \ldots, q_{n}\right\rangle^{\text {in }}={ }^{\text {in }}\left\langle q_{1}^{\prime}, \ldots, q_{m}^{\prime}|S| q_{1}, \ldots, q_{n}\right\rangle^{\text {in }}
$$

where $q_{i}$ and $q_{j}^{\prime}$ label the momenta of the $i n$-going and out-going set of particles. Since in the remote past $t \rightarrow-\infty$ the interaction is not present yet, the asymptotic in states coincide with the unperturbed ones

$$
\left|q_{1}, \ldots, q_{n}\right\rangle^{i n}=\left|q_{1}, \ldots, q_{n}\right\rangle_{0}^{i n}
$$

\footnotetext{
${ }^{6}$ We will comment on the more general case in Section 5 when we will consider the Ising model.
} 
As usual, the scattering operator $S$ appearing in (2.5) can be obtained as the limit

$$
S=\lim _{t \rightarrow+\infty} U(t,-t)
$$

of the time evolution operator $U\left(t, t_{0}\right)$, which is the solution of the equation

$$
i \frac{d}{d t} U\left(t, t_{0}\right)=H U\left(t, t_{0}\right), \quad U\left(t_{0}, t_{0}\right)=1,
$$

where $H=H_{0}+H_{I}$ denotes the Hamiltonian associated to the theory (2.4). Following the standard quantum mechanical procedure (see, for instance [21]), the operator $U$ can be factorised as $U=U_{0} U_{I}$, where $U_{0}$ and $U_{I}$ are solutions of eq. (2.8) with $H$ replaced by $H_{0}$ and $\tilde{H}_{I}(t)=U_{0}^{-1} H_{I} U_{0}$, respectively. Then, we can write the scattering operator of the theory (2.4) as $S=S_{0} S_{I}$, where $S_{0}=\lim _{t \rightarrow+\infty} U_{0}(t,-t)$ is the unperturbed and exactly known scattering matrix

$S_{0}\left\{q_{1}, \ldots, q_{n} \rightarrow q_{1}^{\prime}, \ldots, q_{m}^{\prime}\right\}={ }_{0}^{o u t}\left\langle q_{1}^{\prime}, \ldots, q_{m}^{\prime} \mid q_{1}, \ldots, q_{n}\right\rangle_{0}^{i n}={ }_{0}^{i n}\left\langle q_{1}^{\prime}, \ldots, q_{m}^{\prime}\left|S_{0}\right| q_{1}, \ldots, q_{n}\right\rangle_{0}^{i n}$

while $S_{I}$ has the usual formal representation

$$
S_{I}=\lim _{t \rightarrow+\infty} U_{I}(t,-t)=T \exp \left(i A_{I}[\Psi]\right)
$$

The scattering amplitude is therefore given by

$$
\begin{aligned}
& { }^{\text {out }}\left\langle q_{1}^{\prime}, \ldots, q_{m}^{\prime} \mid q_{1}, \ldots, q_{n}\right\rangle^{\text {in }}={ }_{0}^{\text {out }}\left\langle q_{1}^{\prime}, \ldots, q_{m}^{\prime}\left|T \exp \left(-i \lambda \int d^{2} x \Psi(x)\right)\right| q_{1}, \ldots, q_{n}\right\rangle_{0}^{\text {in }}= \\
& =\sum_{k=0}^{+\infty} \frac{(-i \lambda)^{k}}{k !} \int d^{2} x_{1} \ldots d^{2} x_{k}{ }_{0}^{\text {out }}\left\langle q_{1}^{\prime}, \ldots, q_{m}^{\prime}\left|T\left(\Psi\left(x_{1}\right) \ldots \Psi\left(x_{k}\right)\right)\right| q_{1}, \ldots q_{n}\right\rangle_{0}^{\text {in }}, \quad
\end{aligned}
$$

where (2.9) has been used in order to absorb the factor $S_{0}$.

In ordinary lagrangian perturbation theory based on free theories, the computation of scattering amplitudes would now proceed through the use of creation and annihilation operators and Wick's theorem, finally leading to the diagrammatic expansion characteristic of Feynman covariant perturbation theory. Although this approach cannot be generally followed here (because we might not know, in general, a local lagrangian formulation of the theory associated to $\mathcal{A}_{0}$ ), the knowledge of the exact solution of its dynamics, in the form specified at the beginning of this section, naturally suggests to compute 
the scattering amplitudes (2.11) within a framework quite analogous to that of ordinary time-dependent perturbation theory in quantum mechanics. In other words, we initially insert between the operators $\Psi\left(x_{l}\right)$ and $\Psi\left(x_{l+1}\right)(l=1, \ldots, k-1)$ in the second line of (2.11) a sum over a complete set of asymptotic states of the unperturbed theory

$$
\sum_{n}|n\rangle_{0}^{\text {in in }}\left\langle n\left|=1=\sum_{n}\right| n\right\rangle_{0}^{\text {out }} \underset{0}{\text { out }}\langle n|
$$

with $|n\rangle$ denoting an asymptotic state containing $n$ on-shell particles. Then, the integrations on the space coordinates in (2.11) are immediately performed and lead to delta functions constraining the total momentum of the intermediate states to coincide with that of the initial and final states. In doing the integrations on the time variables, the time ordering prescription gives rise in this case to the appearence of energy denominators so that one ends up with the following expression

$$
\begin{aligned}
& { }^{\text {out }}\left\langle q_{1}^{\prime}, \ldots, q_{m}^{\prime} \mid q_{1}, \ldots, q_{n}\right\rangle^{\text {in }}={ }_{0}^{\text {out }}\left\langle q_{1}^{\prime}, \ldots, q_{m}^{\prime} \mid q_{1}, \ldots, q_{n}\right\rangle_{0}^{\text {in }}+ \\
& +(2 \pi)^{2} \delta^{(2)}\left(\sum_{j=1}^{m} q_{j}^{\prime}-\sum_{j=1}^{n} q_{j}\right)\left\{-i \lambda \underset{0}{\text { out }}\left\langle q_{1}^{\prime}, \ldots, q_{m}^{\prime}|\Psi(0)| q_{1}, \ldots, q_{n}\right\rangle_{0}^{\text {in }}+\right. \\
& +\frac{1}{2 \pi i} \sum_{k=2}^{+\infty}(2 \pi \lambda)^{k} \sum_{n_{1}} \ldots \sum_{n_{k-1}}\left[\frac{\delta\left(Q-P_{1}\right) \ldots \delta\left(Q-P_{k-1}\right)}{\left(E-E_{1}+i \epsilon\right) \ldots\left(E-E_{k-1}+i \epsilon\right)} \times\right. \\
& \left.\left.\times{ }_{0}^{\text {out }}\left\langle q_{1}^{\prime}, \ldots, q_{m}^{\prime}|\Psi(0)| n_{1}\right\rangle_{0} \ldots{ }_{0}\left\langle n_{k-1}|\Psi(0)| q_{1}, \ldots, q_{n}\right\rangle_{0}^{i n}\right]\right\}
\end{aligned}
$$

where $E$ and $E_{i}\left(Q\right.$ and $\left.P_{i}\right)$ denote the total energy (momentum) of the initial state and of the $i$-th intermediate state, respectively, and each intermediate sum can be equivalently taken either on the basis of the in states or on that of out states. Since the matrix elements between asymptotic states of the perturbing operator $\Psi(x)$ are supposed to be known, the scattering amplitudes in the quantum field theory (2.4) are in principle computable quantities, order by order in the coupling constant $\lambda$. The above expansion over intermediate states must be contrasted with the usual formalism of covariant perturbation theory in which both energy and momentum are conserved in the internal lines of Feynman diagrams where, however, such lines correspond to off-shell particles.

As it is, the above formula $(2.13)$ is not completely correct though. In fact, the new interaction changes both the vacuum energy density and the mass of the particles. Hence, we have to refine the action $\mathcal{A}_{I}$ by introducing some counterterms to take properly into 
account the correct normalisation of the states. We require the validity of the following normalisation conditions for any value of the coupling constant: the normalisation of the vacuum state

$$
\langle 0 \mid 0\rangle={ }_{0}\langle 0 \mid 0\rangle_{0}=1
$$

and the normalisation of the one-particle states

$$
{ }^{\text {out }}\left\langle q^{\prime} \mid q\right\rangle^{\text {in }}={ }_{0}^{\text {out }}\left\langle q^{\prime} \mid q\right\rangle_{0}^{\text {in }}=2 \pi E \delta\left(q^{1}-q^{1}\right)
$$

The two above conditions should be enforced order by order in perturbation theory when using (2.13) to compute the vacuum to vacuum and the one-particle amplitudes.

Equation (2.14) leads to subtract a constant term $\delta \mathcal{E}_{v a c}(\lambda)$ from the interaction density. This extra term obviously measures the variation of the vacuum energy density under the effect of the perturbation. This variation is usually ignored in lagrangian perturbation theory where the prescription of disregarding the disconnected vacuum bubble diagrams is adopted. We keep track of it here because it is a measurable quantity for the class of models we are considering in this paper.

The correct one-particle normalisation may be obtained by introducing a 'mass' term operator in the interaction density. This operator, denoted here by $O^{(2)}(x)$, can be defined in terms of its (unperturbed) Form Factors, given by

$$
F_{n}^{O^{(2)}}={ }_{0}\left\langle 0\left|O^{(2)}(0)\right| q_{1}, \ldots, q_{n}\right\rangle_{0}^{i n}=\delta_{n, 2}
$$

With this definition, the coefficient in front of the operator $O^{(2)}(x)$ in the interaction density has to be determined by imposing eq. (2.15) order by order in the coupling $\lambda$ and plays the role of a mass counterterm $\delta m^{2}(\lambda)$.

In summary, the correct formula for the scattering amplitude is given by

$$
\begin{aligned}
& { }^{\text {out }}\left\langle q_{1}^{\prime}, \ldots, q_{m}^{\prime} \mid q_{1}, \ldots, q_{n}\right\rangle^{\text {in }}={ }_{0}^{\text {out }}\left\langle q_{1}^{\prime}, \ldots, q_{m}^{\prime} \mid q_{1}, \ldots, q_{n}\right\rangle_{0}^{\text {in }}+ \\
& -i(2 \pi)^{2} \delta^{(2)}\left(\sum_{j=1}^{m} q_{j}^{\prime}-\sum_{j=1}^{n} q_{j}\right) \times
\end{aligned}
$$

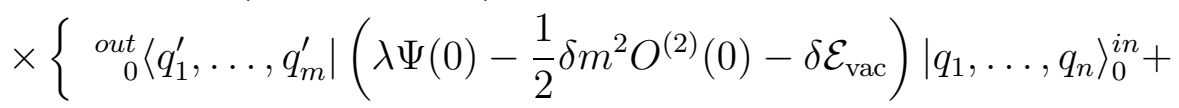

$$
\begin{aligned}
& +\sum_{n_{1}} \frac{2 \pi \delta\left(Q-P_{1}\right)}{\left(E-E_{1}+i \epsilon\right)}{ }_{0}^{\text {out }}\left\langle q_{1}^{\prime}, \ldots, q_{m}^{\prime}\left|\left(\lambda \Psi(0)-\frac{1}{2} \delta m^{2} O^{(2)}(0)-\delta \mathcal{E}_{\text {vac }}\right)\right| n_{1}\right\rangle_{0} \times \\
& \left.{ }_{0}\left\langle n_{1}\left|\left(\lambda \Psi(0)-\frac{1}{2} \delta m^{2} O^{(2)}(0)-\delta \mathcal{E}_{\mathrm{vac}}\right)\right| q_{1}, \ldots, q_{n}\right\rangle_{0}^{i n}+\ldots\right\} .
\end{aligned}
$$


Let us remark that the above expansion appears as the most physical one since it deals with the true physical degrees of freedom of the problem. However, as usual in quantum field theory appearences of divergent contributions are expected when the above formula is applied beyond the first perturbative order. A general discussion of such divergences and of the renormalisation procedure which must be adopted to deal with the infinities, seems to be an interesting open problem in the unconventional setting we are considering. In fact, it must be taken into account that for the perturbations of the interacting theories we are interested in, the perturbing operator $\Psi(x)$ has in general non-vanishing matrix elements on all the asymptotic states. This means that a resummation of an infinite number of terms is required at any perturbative order beyond the first. The general aspects of this problem will not however be further investigated in this paper. Rather, we will concentrate our attention here on the first order approximation in order to study the effects induced on an integrable model of two-dimensional quantum field theory by a small perturbation which breaks the integrability.

\section{Perturbing Two-dimensional Integrable Models}

In this section we apply the perturbative scheme exposed in the previous section to the case in which the term $\mathcal{A}_{0}$ in the euclidean version of the action (2.4) corresponds to an integrable deformation of a two-dimensional conformal field theory through a relevant operator $\Phi(x)$, i.e.

$$
\mathcal{A}_{0}=\mathcal{A}_{C F T}+g \int d^{2} x \Phi(x) .
$$

Before proceeding in our analysis, a brief review of the main properties of the integrable relativistic field theories on which we base our sequent considerations seems in order.

\subsection{Basic Features of Integrable Theories}

Integrable theories are characterized by the existence of an infinite number of integrals of motion. This circumstance gives the possibility of solving them along completely nonperturbative methods. The first consequence of the infinite number of conserved currents is that the scattering processes in the integrable theory are completely elastic, i.e. the 
final state contains the same number of particles with the same momenta of the initial one: the only results of the scattering processes are then a possible exchange of the "color" quantum numbers between particles in the same mass multiplet together with the elastic phase shifts associated (semi-classically) to the time delays of the wave packets [1]. Moreover, in these cases the S-matrix is completely factorised into the product of the two particle scattering amplitudes defined by

$$
{ }_{0}^{\text {out }}\left\langle c\left(\theta_{3}\right) d\left(\theta_{4}\right) \mid a\left(\theta_{1}\right) b\left(\theta_{2}\right)\right\rangle_{0}^{i n}=(2 \pi)^{2} \delta\left(\theta_{1}-\theta_{3}\right) \delta\left(\theta_{2}-\theta_{4}\right) S_{a b}^{c d}\left(\theta_{1}-\theta_{2}\right)
$$

where the rapidity variable $\theta_{i}$ parameterises the on-mass shell energy and momentum of the particles as $\left(p_{i}^{0}, p_{i}^{1}\right)=\left(m_{i} \cosh \theta_{i}, m_{i} \sinh \theta_{i}\right)$. Since the Mandelstam variable $s$, given by

$$
s_{a b}\left(\theta_{1}-\theta_{2}\right)=\left(p_{a}\left(\theta_{1}\right)+p_{b}\left(\theta_{2}\right)\right)^{2}=m_{a}^{2}+m_{b}^{2}+2 m_{a} m_{b} \cosh \left(\theta_{1}-\theta_{2}\right)
$$

is the only independent relativistic invariant in the process, the scattering amplitude depends on the rapidity difference only. Once combined with the general principles of analyticity, unitarity and crossing symmetry, the elasticity and factorisation properties usually lead to the exact determination of the $S$-matrix in integrable models (see for instance [1-9]).

The second important characteristic of the two-dimensional relativistic integrable models is that, in addition to the $S$-matrix, we can also determine exactly the matrix elements of the local operators $\mathcal{O}(x)$ of the theory on the asymptotic states, i.e.

$$
b_{1} \ldots b_{m} F_{a_{1} \ldots a_{n}}^{\mathcal{O}}\left(\theta_{1}^{\prime}, \ldots, \theta_{m}^{\prime} \mid \theta_{1}, \ldots, \theta_{n}\right) \equiv \underset{0}{\text { out }}\left\langle b_{1}\left(\theta_{1}^{\prime}\right) \ldots b_{m}\left(\theta_{m}^{\prime}\right)|\mathcal{O}(0)| a_{1}\left(\theta_{1}\right) \ldots a_{n}\left(\theta_{n}\right)\right\rangle_{0}^{\text {in }}
$$

We can always restrict our attention to those matrix elements with no particle on the left side, the so-called Form Factors (FF)

$$
F_{a_{1} \ldots a_{n}}^{\mathcal{O}}\left(\theta_{1}, \ldots, \theta_{n}\right)={ }_{0}\left\langle 0|\mathcal{O}(0)| a_{1}\left(\theta_{1}\right) \ldots, a_{n}\left(\theta_{n}\right)\right\rangle_{0}^{i n}
$$

In fact, the generic matrix element (3.4) can be obtained in terms of the Form Factors through the analytic continuation

$$
\begin{aligned}
& b_{1} \ldots b_{m} F_{a_{1} \ldots a_{n}}^{\mathcal{O}}\left(\theta_{1}^{\prime}, \ldots, \theta_{m}^{\prime} \mid \theta_{1}, \ldots, \theta_{n}\right)=F_{\bar{b}_{1} \ldots \bar{b}_{m} a_{1} \ldots a_{n}}^{\mathcal{O}}\left(\theta_{1}^{\prime}+i \pi, \ldots, \theta_{m}^{\prime}+i \pi, \theta_{1}, \ldots, \theta_{n}\right) \\
& \quad+\text { disconnected parts }
\end{aligned}
$$


where the bar denotes the charge-conjugated particles. The "disconnected parts" in the right hand side of (3.6) obviously appear when some of the primed rapidities coincide with the unprimed ones (this point is discussed in more detail in appendix B).

As well known, the computation of the Form Factors can be performed once the exact $S$-matrix and the bound state structure of the theory are known. In fact, the monodromy properties of the FF are constrained by the so-called Watson equations, given by

$$
\begin{gathered}
F_{a_{1} \ldots a_{i} a_{i+1} \ldots a_{n}}^{\mathcal{O}}\left(\theta_{1}, \ldots, \theta_{i}, \theta_{i+1}, \ldots, \theta_{n}\right)= \\
=S_{a_{i} a_{i+1}}^{b_{i} b_{i+1}}\left(\theta_{i}-\theta_{i+1}\right) F_{a_{1} \ldots b_{i+1} b_{i} \ldots a_{n}}^{\mathcal{O}}\left(\theta_{1}, \ldots, \theta_{i+1}, \theta_{i}, \ldots, \theta_{n}\right), \\
F_{a_{1} a_{2} \ldots a_{n}}^{\mathcal{O}}\left(\theta_{1}+2 \pi i, \theta_{2}, \ldots, \theta_{n}\right)=F_{a_{2} \ldots a_{n} a_{1}}^{\mathcal{O}}\left(\theta_{2}, \ldots, \theta_{n}, \theta_{1}\right),
\end{gathered}
$$

whereas their analytic structure is closely related to the underlying pattern of singularities induced by the (multi)-scattering processes [11, 12, 13] and is ruled by a set of recursive equations. The simplest of these equations] are obtained by the residue of the $\mathrm{FF}$ at the kinematical poles of particle-antiparticle singularities of relative rapidity $\theta=i \pi[13$

$$
-i \lim _{\tilde{\theta} \rightarrow \theta} F_{\bar{a} a a_{1} \ldots a_{n}}^{\mathcal{O}}\left(\tilde{\theta}+i \pi, \theta, \theta_{1}, \ldots, \theta_{n}\right)=\left(1-\prod_{1}^{n} S_{a a_{i}}\left(\theta-\theta_{i}\right)\right) F_{a_{1} \ldots a_{n}}^{\mathcal{O}}\left(\theta_{1}, \ldots, \theta_{n}\right) .
$$

Additional recursive equations are induced both by the bound-state poles and higherorder poles due to multi-scattering virtual processes [11, 12, 13]. Finally, the asymptotic behaviour of the FF for very large values of the rapidities can be controlled according to a simple criterion proposed in [11]: denoted by $x_{\mathcal{O}}$ the scaling dimension of the operator $\mathcal{O}(x)$ and by $y_{\mathcal{O}}$ the real quantity defined by

$$
\lim _{\left|\theta_{i}\right| \rightarrow \infty} F_{a_{1} \ldots a_{n}}^{\mathcal{O}}\left(\theta_{1}, \ldots, \theta_{n}\right) \sim e^{y_{\mathcal{O}}\left|\theta_{i}\right|}
$$

we have

$$
y_{\mathcal{O}} \leq \frac{x_{\mathcal{O}}}{2}
$$

The equations and the constraints which we have briefly illustrated above prove in general sufficient to determine the FF of the local operators of the integrable theories (see, for instance [11-20]).

\footnotetext{
${ }^{7}$ For simplicity we quote the formula corresponding to the case of diagonal scattering.
} 


\subsection{First Order Perturbation Theory}

Let us now deform the integrable action (3.1) by adding to it the relevant operator $\Psi(x)$. Both the operators $\Phi(x)$ and $\Psi(x)$ are assumed to be scalar and relevant, with their scaling dimensions denoted by $x_{\Phi}$ and $x_{\Psi}$, respectively. The theory (2.4) depends in this case on the two dimensionful couplings constants $g$ and $\lambda$. Since $g \sim M^{2-x_{\Phi}}$ and $\lambda \sim M^{2-x_{\Psi}}$, where $M$ is a mass scale, we can decide to use $g$ as dimensionful parameter of the theory and the dimensionless combination

$$
\chi \equiv \lambda g^{-\frac{2-x_{\Psi}}{2-x_{\Phi}}}
$$

as a label of the different Renormalization Group trajectories which originate from the fixed point at $g=\lambda=0$ (see Fig. 2). For example, if $N(\chi)$ denotes the number of stable particles in the spectrum of the theory, their masses may be expressed as

$$
m_{a}(g, \chi)=\mathcal{C}_{a}(\chi) g^{\frac{1}{2-x_{\Phi}}}, \quad a=1,2, \ldots, N(\chi)
$$

where $\mathcal{C}_{a}(\chi)$ is an amplitude which characterises the whole trajectory. Similarly, the vacuum energy density can be written as

$$
\mathcal{E}_{\mathrm{vac}}(g, \chi)=\mathcal{D}(\chi) g^{\frac{2}{2-x_{\Phi}}}
$$

Dimensionless quantities, as for instance mass ratios, only depend on $\chi$ and therefore they do not vary along the trajectories of the Renormalization Group.

Once the new interaction $\lambda \int d^{2} x \Psi(x)$ is switched on in the action, the integrability characterising the unperturbed theory is generally lost and the S-matrix becomes extremely more complicated. Inelastic processes of particle production are no longer forbidden and the analytic structure of the scattering amplitudes will present additional cuts due to the higher thresholds. In particular, their expression is no longer factorized into the sequence of two-body scattering amplitudes and, even in elastic channels, the only surviving restriction on the final momenta comes from energy-momentum conservation.

\footnotetext{
${ }^{8}$ For the sake of simplicity of notation, we assume that no other interaction term is generated by renormalisation effects (this is the case of the two models which we will explicitely discuss in this paper), although the first order results of this section do not actually depend on this assumption.
} 
The knowledge of the matrix elements (3.4) of the perturbing field $\Psi(x)$ ensures the possibility to compute perturbatively the amplitudes of the inelastic processes as well as the corrections to the elastic ones. To the first order in $\lambda$ and with an obvious extension of the notation, equation (2.17) reads

$$
\begin{aligned}
{ }^{\text {out }} & \left\langle b_{1}\left(q_{1}^{1}\right) \ldots b_{m}\left(q_{m}^{1}\right) \mid a_{1}\left(p_{1}^{1}\right) \ldots a_{n}\left(p_{n}^{1}\right)\right\rangle^{\text {in }} \simeq \\
& \simeq \delta_{m n}{ }^{\text {out }}\left\langle b_{1}\left(q_{1}^{1}\right) \ldots b_{n}\left(q_{n}^{1}\right) \mid a_{1}\left(p_{1}^{1}\right) \ldots a_{n}\left(p_{n}^{1}\right)\right\rangle_{0}^{\text {in }}+ \\
- & i \delta^{2}\left(\sum_{k=1}^{n} p_{k}^{\mu}-\sum_{k=1}^{m} q_{k}^{\mu}\right){ }_{0}^{\text {out }}\left\langle b_{1}\left(q_{1}^{1}\right) \ldots b_{n}\left(q_{m}^{1}\right)\right| \lambda(\Psi(0)+ \\
& \left.-\frac{1}{2} \sum_{a, b=1}^{N} \delta M_{a b}^{2} O_{a b}^{(2)}(0)-\delta \mathcal{E}_{\mathrm{vac}}\right)\left|a_{1}\left(p_{1}^{1}\right) \ldots a_{n}\left(p_{n}^{1}\right)\right\rangle_{0}^{\text {in }} .
\end{aligned}
$$

The "mass operator" $O_{a b}^{(2)}(x)$ is defined assigning its form factors, which with an obvious generalization of eq.(2.16), are given by

$$
F_{a_{1} \ldots a_{n}}^{O_{a b}^{(2)}}\left(\theta_{1}, \ldots, \theta_{n}\right)=\delta_{n 2} \delta_{a a_{1}} \delta_{b a_{2}}
$$

The first order corrections to the masses of the particles and to the vacuum energy density are then obtained imposing the conditions (2.15) and (2.14). As a result, we have

$$
\begin{gathered}
\delta M_{\bar{b} a}^{2} \simeq 2 \lambda F_{\bar{b} a}^{\Psi}(i \pi, 0) \delta_{m_{a} m_{b}}, \\
\delta \mathcal{E}_{\mathrm{vac}} \simeq \lambda\left[0\langle 0|\Psi| 0\rangle_{0}\right] .
\end{gathered}
$$

Care must be however taken when using the rapidity parameterisation in eq. (3.14). Let's illustrate this point by considering the first order correction to some elastic process $a b \rightarrow c d$. In the unperturbed theory, this process is characterised by the scattering amplitude $S_{a b}^{c d}(\theta)$, where $\theta=\theta_{1}-\theta_{2}$ denotes the rapidity difference of the colliding particles. In two dimensions, the momenta of the particles in a two-body elastic collision are individually conserved even in absence of integrability, so that the general elastic amplitude $S_{a b}^{c d}(\theta, \chi)$ can be introduced through the relation

$$
{ }^{\text {out }}\left\langle c\left(\theta_{1}\right) d\left(\theta_{2}\right) \mid a\left(\theta_{3}\right) b\left(\theta_{4}\right)\right\rangle^{\text {in }}=(2 \pi)^{2} \delta\left(\theta_{1}-\theta_{3}\right) \delta\left(\theta_{2}-\theta_{4}\right) S_{a b}^{c d}\left(\theta_{1}-\theta_{2}, \chi\right)
$$

Notice, however, that away from the integrable direction (i.e. $\chi=0$ ), the scattering amplitude $S_{a b}^{c d}(\theta, \chi)$ is no longer a meromorphic function of $\theta$ since the opening of inelastic 
channels induces an additional analytic structure. When we compute the correction to $S_{a b}^{c d}(\theta, \chi)$ around $\chi=0$, we must take into account that, since the total energy of the colliding system is fixed, the variation in the masses given by eq. (3.16) induces a corresponding change in the rapidity difference expressed by

$$
\delta \theta \simeq-\frac{m_{a} \delta m_{a}+m_{b} \delta m_{b}+\left(m_{b} \delta m_{a}+m_{a} \delta m_{b}\right) \cosh \theta}{m_{a} m_{b} \sinh \theta} .
$$

Then the correction to the amplitude can be decomposed as

$$
\delta S_{a b}^{c d}(\theta, \chi)=\frac{\partial S_{a b}^{c d}(\theta)}{\partial \theta} \delta \theta+\left.\frac{\partial S_{a b}^{c d}(\theta, \chi)}{\partial \chi}\right|_{\chi=0} \delta \chi
$$

The first order result for this quantity is obtained by using formula (3.14). Taking into account the cancellation occurring between the disconnected parts in eq. (3.6) and the contributions of the counterterms, one finally obtains

$$
\delta S_{a b}^{c d}(\theta, \chi) \simeq-i \lambda \frac{F_{\bar{c} \bar{d} a b}^{\Psi}(\theta)}{m_{a} m_{b} \sinh \theta},
$$

where

$$
F_{\bar{c} \bar{d} a b}^{\Psi}\left(\theta_{1}-\theta_{2}\right) \equiv F_{\bar{c} \bar{d} a b}^{\Psi}\left(\theta_{1}+i \pi, \theta_{2}+i \pi, \theta_{1}, \theta_{2}\right)
$$

The right hand side of (3.21) employes the expression of the Form Factor at very special values of the rapidity variables. According to eq. (3.8), the Form Factors present pole singularities whenever the rapidities of a particle-antiparticle pair differ by $i \pi$ and, in fact, these kinematical poles are often explicitly inserted into the denominator of their parameterization. Apart from a term encoding the monodromy properties, this parameterization may be written as $\mathcal{Q} / \mathcal{D}$ where both $\mathcal{Q}$ and $\mathcal{D}$ are polynomials in the variables $\cosh \theta_{i j}$, the denominator being fixed by the pole structure whereas the numerator determined by means of the residue equations, as for instance those of eq. (3.8). From the finiteness of the left hand side of eq. (3.21), we expect therefore that the " $i \pi$ singularities" of the denominator of the Form Factors $F_{\bar{a} \bar{b} a b}^{\Psi}\left(\theta_{1}, \theta_{2}, \theta_{3}, \theta_{4}\right)$ should be cancelled by the polynomial $\mathcal{Q}$, once evaluated at the specific rapidity configuration of eq. (3.22). This prediction will be explicitly checked in the next section and should hold in general whenever the perturbing operator is local with respect to the fields which create the particles 
in the unperturbed theoryf?

Eqs. (3.16), (3.17) and (3.21) are the main results of this section. The best use of these formulas is to get rid of the explicit dependence on the normalisation of the perturbing operator by defining universal quantities, as for instance ratios of the mass shifts. Hence, under the validity of the linear approximation, all the universal quantities of nonintegrable field theories can be entirely expressed in terms of the Form Factors of the integrable ones. Comparison of the theoretical predictions with their numerical determinations will be presented in the next sections of the paper.

It is particularly instructive to specialise the above discussion to the "trivial" case in which the perturbing operator $\Psi(x)$ coincides with the operator $\Phi(x)$ that defines the initial integrable theory. In this case of course the physics should be invariant since the result of the additional perturbation simply corresponds to a shift of the coupling constant of the original integrable model by an amount $\delta g=\lambda$. The variations of the masses of the particles and of the vacuum energy density corresponding to such a shift can be directly computed from eqs. (3.11) and (3.12), respectively. On the other hand, we can also apply our general formulas (3.16) and (3.17) to estimate the first order corrections. The two different routes coincide as long as the following identities are valid

$$
\begin{aligned}
& F_{\bar{a} a}^{\Theta}(i \pi, 0)=2 \pi m_{a}^{2}, \\
& \mathcal{E}_{\text {vac }}=\frac{1}{4 \pi}\langle 0|\Theta| 0\rangle,
\end{aligned}
$$

where $\Theta(x)=2 \pi g\left(2-x_{\Phi}\right) \Phi(x)$ is the trace of the energy-momentum tensor for the trajectory $\chi=0$. The above two relationships are indeed true and can be easily derived by other means. However, it is interesting to notice that their validity emerges in this context for the role of consistency equations which they play. Obviously, by the same token we can generate an infinite number of identities involving Form Factors of the original integrable field theory by considering higher multi-particle scattering processes. For instance, next to (3.23), a new identity is obtained by comparing eq. (3.20) with eq. (3.21): since $\chi$ is constant in the case we are considering, we have

$$
\frac{\partial S_{a b}^{c d}(\theta)}{\partial \theta}=-\frac{1}{2 \pi i} \frac{F_{\bar{c} \bar{d} a b}^{\Theta}(\theta)}{s_{a b}(\theta)} .
$$

\footnotetext{
${ }^{9}$ We defer to the section devoted to the Ising model the discussion of the case in which this condition is not fulfilled.
} 
This identity provides a simple and unique way to normalise the four-particle Form Factors of the stress-energy tensor. It may be then particularly useful in the study of massless field theories where the first relationship in eq. (3.23) cannot be used for this purpose. For instance, it is easy to check that eq. (3.24) applies to the Form Factor of the massless model considered in ref. [19].

It is also obvious that the first order inelastic amplitudes computable by formula (3.14) must vanish identically when we choose $\Psi(x)=\Phi(x)$. This is ensured by the fact that the form factors of the stress-energy tensor $F_{a_{1} \ldots a_{n}}^{\Theta}\left(\theta_{1}, \ldots, \theta_{n}\right)$ factorise the term $P_{\mu} P^{\mu}$, with $P^{\mu}=\sum_{i=1}^{n} p_{i}^{\mu}$ denoting the total energy-momentum of the set of particles. Since $p_{i}^{\mu} \rightarrow-p_{i}^{\mu}$ when the $i-$ th particle is crossed from the initial to the final state, $P^{\mu}$ is zero for a set of particles entering a physical scattering process. Only in the case of elastic scattering, the zeros coming from the factor $P_{\mu} P^{\mu}$ are cancelled by the kinematical poles and therefore relations analogous to eq. (3.24) are obtained.

\section{Non-Integrable Deformations of the Minimal Model $M_{(2,7)}$}

As already indicated in the introduction, the main idea of using a perturbative expansion based on the Form Factors of integrable theories is because we expect that this kind of series should be capable of approximating the dynamics of the non-integrable field theories close to the integrable ones better than any other approach. One of the physical reasons for this expectation is that the integrable field theories should provide from the start the right multi-particle basis in the Hilbert space of the perturbed, non-integrable ones: hence, the differences between their physical properties are presumed to be small and calculable. Said in mathematical terms, such perturbative expansion should be particularly significant since the exact expression of the Form Factor already corresponds to a resummation of an infinite number of terms originating from the action (1.2). However, the final conclusion on the efficiency of the Form Factor perturbative approach should come from some explicit and direct comparison with a set of data obtained from other sources. This is what we are going to accomplish in this section as well as in the next one. 
As our first example, we will discuss the scaling region around the fixed point described by the non-unitarity minimal model $M_{(2,7)}$. This model is particularly appealing for its simplified dynamics whereby the significant physical effects we are looking for will not be masked by other additional complications.

The minimal conformal model $M_{(2,7)}$ has only two primary operators, $\Phi_{1,3}$ and $\Phi_{1,2}$, both of them relevant with scaling dimensions given by $-6 / 7$ and $-4 / 7$ respectively [28]. The perturbations of the conformal action either by the operator $\Phi_{1,3}$ or by the operator $\Phi_{1,2}$ are both known to be, separately, integrable [0], 8]. In their massive phase, both perturbations have two stable massive particles denoted by $A_{1}(\theta)$ and $A_{2}(\theta)$, with a mass ratio and a scattering matrix which depend, however, on the integrable direction considered. The exact two-particle elastic $S$-matrix and other relevant information about the two different integrable deformations are summarized in the following table

\begin{tabular}{|c|c|}
\hline$\Phi_{1,3}$ deformation & $\Phi_{1,2}$ deformation \\
\hline$S_{11}(\theta)=\left(\frac{2}{5}\right)$ & $S_{11}(\theta)=\left(\frac{2}{3}\right)\left(\frac{1}{9}\right)\left(-\frac{2}{9}\right)$ \\
$S_{12}(\theta)=\left(\frac{3}{5}\right)\left(\frac{4}{5}\right)$ & $S_{12}(\theta)=\left(\frac{17}{18}\right)\left(\frac{11}{18}\right)$ \\
$S_{22}(\theta)=\left(\frac{4}{5}\right)\left(\frac{2}{5}\right)^{2}$ & $S_{22}(\theta)=\left(\frac{2}{3}\right)\left(\frac{1}{9}\right)\left(\frac{5}{9}\right)$ \\
\hline$\frac{m_{2}}{m_{1}}=2 \cos \frac{\pi}{5}=1.6180 \ldots$ & $\frac{m_{2}}{m_{1}}=2 \cos \frac{\pi}{18}=1.9696 \ldots$ \\
\hline $\mathcal{E}_{v a c}=-\frac{m_{1}^{2}}{8 \sin \frac{2 \pi}{5}}=-0.1314 . . m_{1}^{2}$ & $\mathcal{E}_{v a c}=-\frac{m_{1}^{2}}{8\left(\sin \frac{\pi}{3}+\sin \frac{\pi}{9}-\sin \frac{2 \pi}{9}\right)}=-0.2211 . . m_{1}^{2}$ \\
\hline
\end{tabular}

where

$$
(\alpha) \equiv \frac{\tanh \frac{1}{2}(\theta+i \pi \alpha)}{\tanh \frac{1}{2}(\theta-i \pi \alpha)},
$$

and the bound state poles in the $S$-matrix amplitudes related to the particles $A_{i}$ are identified by the index $i$ placed above the functions $(\alpha)$.

At the present, the $\Phi_{1,3}$ perturbation is the most studied of the two and in particular the Form Factors of the two primary fields are known [14]. This suggests to adopt as our initial euclidean action $\mathcal{A}_{0}$ that relative to the field $\Phi_{1,3}$, i.e.

$$
\mathcal{A}_{0}=\mathcal{A}_{(2,7)}+g \int d^{2} x \Phi_{1,3}(x)
$$


and then add to it the other deformation 10

$$
\mathcal{A}=\mathcal{A}_{0}+\lambda \int d^{2} x \Phi_{1,2}(x)
$$

It is natural to assume that the spectrum associated to the action (4.3) consists for all positive values of the dimensionless parameter $\chi$ of two massive non-degenerate excitations (the validity of this assumption can be directly confirmed by means of a numerical technique that we will mention below). Hence, the QFT described by (4.3) is of the type of isospectral theory discussed in Section 2. This circumstance, together with the relative simplicity of the scattering theory in both integrable directions, makes this theory the ideal playground for testing the Form Factor perturbative scheme. In order to make explicit predictions, we have listed in Appendix $\mathrm{C}$ the matrix elements of the operator $\Phi_{1,2}$ that we need in the sequel.

Let us initially compute the first order correction to the elastic scattering amplitude $S_{11}(\theta)$. According to the general formula (3.21), this is given in terms of the fourparticle Form Factor given by eq. (C.4), computed at specific values of the rapidities. The corresponding particle configuration consists of neutral particles with rapidities differing by $i \pi$, i.e. the $\mathrm{FF}$ is on the resonant configuration ruled by the "kinematical poles" of the factors $\cosh \left(\theta_{k l} / 2\right)$ in the denominator of (C.4). However, the factor $\mathcal{Q}\left(\theta_{i j}\right)$ present in the numerator of the four-particle Form Factor exactly cancels these divergences and produces as a finite result

$$
S_{11}(\theta, \lambda) \simeq \frac{\tanh \frac{1}{2}\left(\theta+i \frac{2 \pi}{5}\right)}{\tanh \frac{1}{2}\left(\theta-i \frac{2 \pi}{5}\right)}-i \frac{\lambda c_{0}}{m_{1}(g, 0)^{2}}\left(32 \sin ^{2} \frac{\pi}{5}\right) \frac{\cosh ^{2} \frac{\theta}{2}}{\sinh \theta} \frac{1+2 \cos \frac{2 \pi}{5} \cosh \theta}{\left(\sinh \theta-i \sin \frac{2 \pi}{5}\right)^{2}} .
$$

The rapidities employed in the above formula are obviously defined on the unperturbed mass-shell condition. The finiteness of the above formula is an explicit demonstration of the cancellation of the kinematical singularities of the FF discussed in the previous section.

\footnotetext{
${ }^{10}$ Throughout this section we will refer to the region of the coupling space of the theory $(4.3)$ in which, within the standard CFT normalisation of the fields, $g$ is real and negative and $\lambda$ is purely imaginary with positive imaginary part. The presence of the imaginary coupling is not surprising in view of the non-unitary nature of the original conformal model.
} 
The correction term in (4.4) exhibits a second order pole at $\theta=2 \pi / 5$. The increased order of the pole signals that the position of the bound state pole in the $\theta$-plane has become in the non-integrable theory a function of the coupling constant $\lambda$. This suggests the reabsorbing of the first order term in (4.4) as a correction to the location of the original simple pole, given now by

$$
\theta=i u=i\left(\frac{2 \pi}{5}+\frac{\lambda c_{0}}{\left(m_{1}^{0}\right)^{2}} 8\left(\cos \frac{2 \pi}{5}+2 \cos \frac{\pi}{5}\right)\right)+O\left(\lambda^{2}\right) .
$$

For the mass of the heaviest particle we then have

$$
\begin{aligned}
m_{2} & =2 m_{1}^{0} \cos \frac{u}{2} \simeq \\
& \simeq 2 m_{1}^{0} \cos \left[\frac{\pi}{5}\left(1-\frac{\lambda c_{0}}{\left(m_{1}^{0}\right)^{2}} 8 \sin \frac{\pi}{5}\left(1+2 \cos ^{2} \frac{2 \pi}{5}\right)\right)\right]= \\
& =m_{2}^{0}\left[1-\frac{\lambda c_{0}}{\left(m_{1}^{0}\right)^{2}} 8 \sin \frac{\pi}{5}\left(1+2 \cos ^{2} \frac{2 \pi}{5}\right)\right],
\end{aligned}
$$

where $m_{i}^{0}$ denote the unperturbed value of the masses. By using eq. (C.3) it is easy to check that the above expression indeed coincides with the value directly obtained from eq. (3.16), i.e.

$$
m_{2}^{2}=\left(m_{2}^{0}\right)^{2}+2 \lambda F_{22}(i \pi)
$$

In addition to this consistency check, a direct test of the theoretical predictions for the variations in the spectrum of the theory under the perturbation can be obtained by the so-called "truncation method" [35]. The basic idea of this approach is to study the theory on an infinitely long strip of width $R$ (the linear spatial volume) with periodic boundary conditions. After choosing a Hilbert space basis of eigenvectors of the ultraviolet conformal Hamiltonian, all the matrix elements of the perturbed Hamiltonian on this basis can be exactly computed. The off-critical spectrum can then be found by numerical diagonalisation on a truncated Hilbert space containing a suitable number of states $\square$. Since the method does not rely in any way on integrability, it can be applied to any perturbation of the conformal point. In particular, for the theory defined by the action (2.4), the energy levels must have the scaling form

$$
E_{i}(R, g, \lambda)=\frac{2 \pi}{R} f_{i}\left(m_{1} R ; \chi\right), \quad i=0,1,2 \ldots .
$$

\footnotetext{
${ }^{11}$ All these steps can be performed by means of the algorithm developped in [36].
} 
At very short distance scales, the critical fluctuations are expected to dominate so that the spectrum must coincide with that of the conformal point given by [29]

$$
E_{i} \simeq \frac{2 \pi}{R}\left(x_{i}-\frac{c}{12}\right), \quad m_{1} R<<1
$$

where $c$ denotes the central charge and $x_{i}$ the scaling dimensions of the scaling fields in the underlying conformal theory. In the infrared limit, on the other hand, one should recover the spectrum of the massive theory on the plane and therefore the energy levels are given by

$$
E_{i} \simeq \mathcal{E}_{v a c}(g, \chi) R+m_{i}(g, \chi), \quad m_{1} R>>1
$$

where the first term takes into account the vacuum bulk energy contribution and $m_{i}$ denotes the mass-gap of the i-th level[ㅍ. The first energy levels in the spectrum of the pure $\Phi_{1,3}$ perturbation of the conformal model $M_{(2,7)}$ obtained by including all conformal states up to level five in the Verma modules are shown in Fig. 3. Starting from the bottom, the first four parallel lines are easily identified as the vacuum energy level, the one-particle energy level of the lowest particle $A_{1}$, the one-particle energy level of the heaviest particle $A_{2}$ and the two-particle threshold energy line, respectively. The remaining levels will be part of the continuum in the infinite volume limit. The measured values of the mass ratio $m_{2}^{0} / m_{1}^{0} \simeq 1.61$ and the vacuum energy density $\mathcal{E}_{\text {vac }}^{0} \simeq-0.13\left(m_{1}^{0}\right)^{2}$ are in good agreement with the theoretical expectations of the corresponding integrable theory. As for other spectra of integrable models [35, 37, 38, 39], several crossings of the energy levels are expected and they are indeed observed for this integrable deformation of the $M_{(2,7)}$ model. When a small $\Phi_{1,2}$ perturbation is switched on (keeping $g$ fixed), the first qualitative effect consists to resolve the degeneracy present at the crossing points, i.e. all energy levels have now the tendency to repel each other. This feature can be

\footnotetext{
12 The above asymptotic expression only applies in the ideal situation where we discard the effects induced by the truncation of the Hilbert space. Although one can also take into account these extra numerical effects and refine consequently the above formulas, the long practice with integrable massive perturbations has shown that as far as the low lying energy levels are concerned, they are not much affected by truncation effects so that they can be obtained with a remarkable accuracy on a large range of $R$ by just including few conformal states in the calculation. This observation obviously helps to speed up the numerical work.
} 
interpreted as the breaking of the initial integrability of the model since it is well known that, in absence of any higher symmetry, two hamiltonian lines cannot generally cross. At a quantitative level, for small values of $\lambda$, both the separation and the slope of the lines vary linearly with it. The first order variations of these quantities are measured to be

$$
\begin{aligned}
& \frac{\delta m_{2}}{\delta m_{1}} \simeq 3.74 \\
& \frac{\delta \mathcal{E}_{v a c}}{\delta m_{1}} \simeq 0.67 m_{1}^{0} .
\end{aligned}
$$

They must be compared with the theoretical predictions for the same quantities obtained by using eqs. (3.16) and (3.17)

$$
\begin{aligned}
& \frac{\delta m_{2}}{\delta m_{1}}=\frac{m_{1}^{0}}{m_{2}^{0}} \frac{F_{22}^{(1,2)}(i \pi)}{F_{11}^{(1,2)}(i \pi)}=2\left(4 \cos \frac{\pi}{5} \sin ^{2} \frac{2 \pi}{5}-1\right)=3.8541 . . \\
& \frac{\delta \mathcal{E}_{v a c}}{\delta m_{1}}=m_{1}^{0} \frac{\left\langle 0\left|\phi_{1,2}\right| 0\right\rangle}{F_{11}^{(1,2)}(i \pi)}=-\frac{\cos \frac{\pi}{5}}{2 \sin \frac{\pi}{5}}=0.68819 . . m_{1}^{0} .
\end{aligned}
$$

The agreement between the theoretical estimates and the measured values is therefore quite satisfactory, the discrepancy being of the same order of the numerical error introduced by truncation effects.

The truncation method obviously allows us to study the spectrum of the theory for any values of the coupling constants $g$ and $\lambda$ and to explicitly test several theoretical assumptions. We have for instance verified the validity of the scaling law (3.11) for the spectrum, which in this theory always consists of two distinct one-particle states whose mass ratio smoothly interpolates between the values $m_{2} / m_{1}=2 \cos \frac{\pi}{5}$ and $m_{2} / m_{1}=2 \cos \frac{\pi}{18}$ (relative to the limits $\chi \rightarrow 0$ and $\chi \rightarrow \infty$, respectively), as shown in Figure 4. Obviously for large values of $\chi$ the physical properties of the model can be no longer theoretically predicted by the first terms of perturbative series based on the $\Phi_{1,3}$ deformation. In particular, to study the model in the limit $\chi \rightarrow \infty$, it is evident that instead of including higher order terms of the perturbative series based on the $\Phi_{1,3}$ deformation, it would be more convenient to make use of the other perturbation theory based on the second integrable operator $\Phi_{1,2}$ deformation. 


\section{The Scaling Region of the Two-Dimensional Ising Model}

Aim of this section is to extract through the Form Factor perturbative techniques some information about the scaling region nearby the critical point of the two-dimensional Ising model described by

$$
\mathcal{A}=\mathcal{A}_{(3,4)}+\tau \int d^{2} x \varepsilon(x)+h \int d^{2} x \sigma(x)
$$

and also to study the model by means of the truncation method. As expressed by (5.1), the most general off-critical realization of the Ising model is given by a perturbation of the simplest unitary minimal model $M_{(3,4)}$ with a linear combination of its two only relevant primary fields, i.e. the energy density $\Phi_{1,3}(x) \equiv \varepsilon(x)$ of scaling dimension $x_{\varepsilon}=1$ and the magnetization $\Phi_{1,2}(x) \equiv \sigma(x)$ of scaling dimension $x_{\sigma}=1 / 8$. The conjugated couplings $\tau$ and $h$ are respectively interpreted as the deviation from the critical temperature $T_{c}$ and a constant magnetic field, with physical dimensions given by $\tau \simeq M$ and $h \simeq M^{15 / 8}$, where $M$ is a mass scale. The Zamolodchikov's counting argument [2] ensures that the theory (5.1) becomes integrable as far as one of the two coupling constants $\tau$ or $h$ is set equal to zero. The integrability of the purely thermal perturbation has been known for long time and can be neatly reformulated in terms of field theory of Majorana fermions [12, 15, 22, 23, 24]. The integrability of the magnetic deformation has been established by Zamolodchikov [2] in the recent past and, for the original features of his findings this result may be regarded as a direct success of the application of field theoretical methods to statistical mechanics.

The action (5.1) defines a one parameter family of field theories which can be labelled by the dimensionless combination $\chi \equiv \tau|h|^{-8 / 15} \in(-\infty,+\infty)$. Previous investigations have shown that the particle content of the model must undergo drastic changes as a function of $\chi$ [25, 26]. As we will see in the following, this prediction can be directly confirmed by the numerical determination of the spectrum for different values of $\chi$ (see Figures 7). The reason for such behaviour of the spectrum will be easily understood after reviewing some exact results obtained for the two integrable directions. 


\subsection{Thermal Deformation}

Consider first the case $h=0$. In the high temperature phase, i.e. $\chi=+\infty$, the theory has an unique vacuum state and the spectrum consists of a single particle $A$ of mass $m=2 \pi \tau$ and multi-particle states theoreof. This is clearly confirmed by the truncation method calculation shown in Figure (7.a): starting from the bottom, the sequence of energy levels is given by the ground state, one-particle level and then all the multiparticle lines of the continuum, the lowest of which corresponding to the two-particle threshold. The massive excitation present in this phase of the model can be equivalently regarded as a free neutral fermion or as an interacting boson (with scattering amplitude $S=-1)$ created by the magnetization operator. Invariance under the spin reversal implies that the "order" ("disorder") field $\sigma(x)(\mu(x))$ couples to the states with an odd (even) number of particles only. The Form Factors for the two operators are given by the unique expression [12, 15]

$$
F_{(n)}\left(\theta_{1}, \ldots, \theta_{n}\right) \equiv\left\langle 0|\mathcal{O}(0)| a\left(\theta_{1}\right) \ldots a\left(\theta_{n}\right)\right\rangle=(i)^{\left[\frac{n}{2}-1\right]} \prod_{i<j}^{n} \tanh \frac{\theta_{i}-\theta_{j}}{2},
$$

with $n$ odd for $\mathcal{O}(x)=\sigma(x)$ and even for $\mathcal{O}(x)=\mu(x)$ and $[\alpha]$ denoting the integer part of $\alpha$.

The situation in the low temperature phase, i.e. $\chi=-\infty$, is easily deduced from the previous one by duality. In fact, the excitations are now created by the disorder operator $\mu(x)$ and must be interpreted as kinks and anti-kinks of mass $m$ interpolating between the two degenerate vacua coming from the spontaneous symmetry breaking of the $Z_{2}$ invariance of the model. The Form Factors of $\sigma(x)$ coincide with those of the operator $\mu(x)$ computed in the high-temperature phase $T>T_{c}$. Hence, the magnetization operator $\sigma(x)$ now couples only to states with even number of excitations. The numerical determination of the spectrum is shown in Figure (7.g). The lowest two lines are clearly the degenerate ground state levels. They approach each other exponentially in the crossover region, $\Delta E \sim \exp (-m R)$, where $m$ is the mass of the kink responsible of the finite-size tunnelling effect between the two vacua. Since periodic boundary conditions were adopted on the strip of width $R$ in the numerical determination of the spectrum, in Figure (7.g) there are no energy levels corresponding to odd number of kink 
states. In particular, the energy line associated to the single kink state is absent and therefore the lowest energy level above the ground state energies is given in this case by the kink-antikink threshold line.

\subsection{Magnetic Deformation}

Far richer is the situation for the other integrable direction obtained when $\tau=0$ and $h \neq 0$, i.e. $\chi=0$. In fact, Zamolodchikov has shown that in this case the spectrum consists of eight different species of self-conjugated particles $A_{i}, i=1, \ldots, 8$ with masses 2

$$
\begin{aligned}
& m_{1}=M(h), \\
& m_{2}=2 m_{1} \cos \frac{\pi}{5}=(1.6180 . .) m_{1}, \\
& m_{3}=2 m_{1} \cos \frac{\pi}{30}=(1.9890 . .) m_{1}, \\
& m_{4}=2 m_{2} \cos \frac{7 \pi}{30}=(2.4048 . .) m_{1}, \\
& m_{5}=2 m_{2} \cos \frac{2 \pi}{15}=(2.9562 . .) m_{1}, \\
& m_{6}=2 m_{2} \cos \frac{\pi}{30}=(3.2183 . .) m_{1}, \\
& m_{7}=4 m_{2} \cos \frac{\pi}{5} \cos \frac{7 \pi}{30}=(3.8911 . .) m_{1}, \\
& m_{8}=4 m_{2} \cos \frac{\pi}{5} \cos \frac{2 \pi}{15}=(4.7833 . .) m_{1},
\end{aligned}
$$

Notice that only the first three particles of the above spectrum lie below the lowest threshold at $2 m_{1}$. The remaining particles are however stable since integrability prevents the possibility of inelastic processes, in particular decay processes. The $S$-matrix describing the interactions between the eight particles was exactly determined in [2]. These results

\footnotetext{
${ }^{13}$ Within the standard CFT normalization of the magnetization operator, obtained by the equation $<\sigma(x) \sigma(0)>=\frac{1}{|x|^{\frac{1}{4}}}(|x| \longrightarrow 0)$, the overall mass scale $M(h)$ has been exactly determined in [10], $M(h)=\mathcal{C} h^{\frac{8}{15}}$ where

$$
\mathcal{C}=\frac{4 \sin \frac{\pi}{5} \Gamma\left(\frac{1}{5}\right)}{\Gamma\left(\frac{2}{3}\right) \Gamma\left(\frac{8}{15}\right)}\left(\frac{4 \pi^{2} \Gamma\left(\frac{3}{4}\right) \Gamma^{2}\left(\frac{13}{16}\right)}{\Gamma\left(\frac{1}{4}\right) \Gamma^{2}\left(\frac{3}{16}\right)}\right)^{\frac{4}{5}}=4.40490858 \ldots
$$
}


were used in refs. [11] to implement the bootstrap equations for the Form Factors of the model and to compute in particular the one and two-particle matrix elements of the operator $\sigma(x)$. As expected from the fact that neither the action nor the $S$-matrix of the model exhibit any internal symmetry, the magnetization operator was found to couple to all the eight particles in the spectrum, namely $F_{i}^{\sigma}=\left\langle 0|\sigma| A_{i}\right\rangle \neq 0$ for $i=1, \ldots, 8$.

The finite size spectrum of the Ising model in a magnetic field at $T=T_{c}$ as obtained with the truncation method is shown in Figure (7.d). Starting from the bottom, five parallel lines are clearly identified in the large volume limit. They correspond to the ground state, the three lightest particles and the first two-particle threshold, respectively. The remaining single particle states are of more difficult identification since they are placed among the higher threshold lines. Notice that they cross in several points the "momentum lines" (i.e. lines corresponding to states containing two or more particles not at rest with respect to each other) converging toward the threshold in the infinite volume limit. Level crossing in the energy spectrum is always related to the presence of symmetries and in the present case strongly supports the integrability of the theory.

\subsection{McCoy-Wu Scenario}

After having discussed individually each deformation, let us consider the more general case described by the action (5.1). Following McCoy and $\mathrm{Wu}$ [25], let us introduce the Fourier transform of the spin-spin correlation function

$$
G\left(p^{2}, \chi\right)=\int d^{2} x e^{i p x}\langle\sigma(x) \sigma(0)\rangle
$$

According to the discussion of the previous subsections, the leading infrared singularities of this function in the complex $p$-plane for $\chi=+\infty, 0,-\infty$ are those shown in Figs. (6.a), (6.d) and (6.g), respectively. Since these three situations correspond to different values of the couplings in the same QFT defined by the action (5.1), it must be possible to interpolate continously between them by moving along the semicircular path drawn in Figure 5. To describe the change of physical properties of the theory along the path, the following scenario has been proposed [25] : starting from the point $a$ in Figure 5, the spin reversal symmetry will be broken as far as a magnetic field $h$ is turned on 
and consequently, even particle thresholds immediately appear, as shown in Fig. 6.b. Moreover moving toward the point $d$, additional poles emerge from unphysical sheets of the Riemann surface through the branch cuts and become physical bound state poles. From Zamolodchikov's solution, we know that at the point $d$ there are precisely three such poles below the first two-particle threshold. Their number however continues to increase when moving toward the negative $\tau$ axis until altogether they coalesce at point $g$ and give rise to the branch cut starting at $2 \mathrm{im}$ in Fig. (6.g). The lowest lines of the numerical spectra corresponding to the values 四 $\chi=+\infty, 1.32,0.16,0,-0.16,-1.32$, $-\infty$ are shown in the sequence of Figures 7 and we will comment on them below.

The coalescence of the poles is quite a striking phenomenon. It was quantitatively discussed in ref. 25] where the knowledge of the $n$-point correlators for the purely thermal Ising model was exploited to study the behaviour at small $h$ of the spin-spin correlation function expressed in the form

$$
\langle\sigma(x) \sigma(0)\rangle_{\text {conn }, h}=\sum_{n=0}^{\infty} \frac{1}{n !} h^{n} \int d^{2} x_{1} \ldots d^{2} x_{n}\left\langle\sigma(x) \sigma(0) \sigma\left(x_{1}\right) \ldots \sigma\left(x_{n}\right)\right\rangle_{\text {conn }, h=0} .
$$

It was found that when the magnetic field is switched on, the two-particle branch cut of Fig. (6.g) breaks up in a sequence of poles located at

$$
\left(2+h^{2 / 3} \gamma_{k}^{2 / 3}\right) i m
$$

where $\gamma_{k}$ are the positive solutions of

$$
J_{\frac{1}{3}}\left(\frac{1}{3} \gamma_{k}\right)+J_{-\frac{1}{3}}\left(\frac{1}{3} \gamma_{k}\right)=0
$$

and $J_{\nu}(x)$ is the Bessel function of order $\nu$. The above values coincide with the eigenvalues of the Schroedinger equation for a particle in a central linear potential. The physical origin of this result is that the presence of a small magnetic field breaks the degeneracy of the two vacua in the low-temperature phase inducing a linear confining potential between the kinks $\amalg^{\boxplus}$. It is clearly evident then that the magnetic field induces a drastic change in the structure of the low-temperature phase of the model: in fact, as soon as the magnetic

\footnotetext{
${ }^{14}$ The quoted values of $\chi$ refer to the standard CFT normalisation of the fields $\varepsilon(x)$ and $\sigma(x)$. For a generic field $\varphi$ of scaling dimension $x$, this is defined by the condition $\langle\varphi(r) \varphi(0)\rangle \longrightarrow r^{-2 x}, r \longrightarrow 0$.

${ }^{15}$ One can easily convince himself of this feature by using a semi-classical analysis of the kink states.
} 
field is switched on, the kink configurations are no longer asymptotic states of the field theory and the original kink-antikink pairs collapse into a sequence of bound states. This happens for any value of the magnetic field $h$, however small. The lightest of these bound states has a mass equal to $2 m+O\left(h^{2 / 3}\right)$, so that the first branch point is now located at $4 i m+O\left(h^{2 / 3}\right)$.

\subsection{Form Factor Perturbation Theory}

Let us now turn to the discussion of the Ising model within the perturbative framework considered in this paper $\square$. Our main interest will be to develop the perturbative picture around the integrable magnetic direction $(\chi=0)$, for which no other approach is presently avalaible. Before doing that, we will however briefly comment on the case $h \sim 0$, distinguishing the two situations $T>T_{c}$ and $T<T_{c}$.

At $T>T_{c}$, the magnetization operator $\sigma(x)$ couples to the odd-particle states only. Hence, the opening of inelastic channels (e.g. the production process $A A \longrightarrow A A A$ ) with the consequent breakdown of integrability can be easily checked at first order in $h$ but the correction to the mass or the variation of the elastic scattering amplitudes occurs instead only at the second order in $h$. The breaking of the integrability implies that the numerical spectrum no longer presents in this case crossing of the energy levels, a feature which, although not always clearly visible in Figs. 7, is however confirmed by the numerical data.

In the low-temperature phase $T<T_{c}$ the spectrum of the theory is known to undergo a qualitative and non-analytic change when the magnetic field is switched on. Although these considerations naturally suggest the failure of any finite order of a perturbative approach to study this phenomenon, it is nevertheless instructive to see how such failure manifests itself in our method. To this aim, it is sufficient to attempt the computation of the correction to the mass of the kink, an excitation which we know no longer exists as an asymptotic state in the perturbed theory. According to the general formula (3.16), the first order correction is proportional to the two-particle form factor of the magnetization

\footnotetext{
${ }^{16}$ For other perturbative studies of the Ising model field theory see refs. [40, 41].
} 
operator computed for a rapidity difference equal to $i \pi$. Since this form factor is equal to $\tanh \frac{\theta_{1}-\theta_{2}}{2}$ (see eq. (5.2)), it has a pole at $\theta_{1}-\theta_{2}=i \pi$ and therefore an unbounded correction is obtained for the mass of the kink. The general theory of Form Factor predicts that such "kinematical" pole is present in the two-particle Form Factor $\left\langle 0|\mathcal{O}(0)| A\left(\theta_{1}\right) \bar{A}\left(\theta_{2}\right)\right\rangle$ only if the operator $\mathcal{O}(x)$ is non-local with respect to the field which creates the particle $A$ (as it is the case for the fields $\sigma(x)$ and $\mu(x)$ in the Ising model). This circumstance suggests therefore that a confinement phenomenon of the type described above for the Ising model has to be expected each time that the perturbing operator and the fields which create the particle excitations in the unperturbed theory are not mutually local. It would be interesting to check this prediction in other statistical models where the integrable spectrum is given by kink excitations.

In the case of the Ising model, the numerical determination of the spectrum confirms the above scenario. From Figure (7.f), we see in fact that the first effect of $h$ consists in the removal of the degeneracy of the lowest as well as of the higher eigenvalues. The two originally degenerate ground state levels have now been splitted into: (i) a unique ground state energy line and (ii) an excited state whose mass gap diverges in the large volume limit $R \longrightarrow \infty$. The degeneracy of the lowest threshold line has also been lifted giving rise to a sequence of one-particle energy levels, the lowest values of which can be checked to be in reasonable agreement with eq. (5.6). By increasing the magnetic field $h$, the above characteristic feature of the spectrum are futher enhanced, as seen by following Figs. (7.e) and (7.f) in reverse order: the divergent energy line of the initial degenerate ground states meets all other lines at smaller values of $R$ (and therefore it decouples faster from the remaining spectrum) whereas the other eigenvalues start to assume the structure and the values predicted by the Zamolodchikov solution for $T=T_{c}$, reproduced in Figure (7.d). Observe that, while the eigenvalues have the typical repulsive behaviour of a non-integrable situation all along the path from the point $f$ to $e$, on the contrary they cross each other once we have reached the integrable situation of the pure magnetic axis. Moreover, the fact that in the pure magnetic case the approach to the asymptotic value of the masses is reached from below can be simply interpreted as the reminiscence of the divergent line coming from the degenerate ground state energies of the low-temperature 
phase.

Let us now analyse more closely and in quantitative terms the field theory defined in the vicinity of the magnetic axis. Perturbation theory around the magnetic axis $\left(T=T_{c}\right.$ with $h \neq 0$ ) obviously requires the knowledge of the Form Factors of the energy operator $\varepsilon(x)$ in the Zamolodchikov field theory involving the eight massive states $A_{i}(i=1, \cdots, 8)$ associated to the pure magnetic model. These Form Factors can be exactly computed by using the same bootstrap approach used in ref. [11] for the magnetization operator. The detailed discussion on the computations of these quantities will be presented elsewhere [31] and here we only quote the results needed for our present purposes

$$
\begin{aligned}
& \langle 0|\varepsilon(0)| 0\rangle=m_{1}, \\
& F_{11}^{\varepsilon}(i \pi)=\left\langle 0|\varepsilon(0)| A_{1}(\theta+i \pi) A_{1}(\theta)\right\rangle=-17.8933 . . m_{1}, \\
& F_{22}^{\varepsilon}(i \pi)=\left\langle 0|\varepsilon(0)| A_{2}(\theta+i \pi) A_{2}(\theta)\right\rangle=-24.9467 . . m_{1}, \\
& F_{33}^{\varepsilon}(i \pi)=\left\langle 0|\varepsilon(0)| A_{3}(\theta+i \pi) A_{3}(\theta)\right\rangle=-53.6799 . . m_{1}, \\
& F_{44}^{\varepsilon}(i \pi)=\left\langle 0|\varepsilon(0)| A_{4}(\theta+i \pi) A_{4}(\theta)\right\rangle=-49.3169 . . m_{1} .
\end{aligned}
$$

The first line of the above relationships should be meant as expressing the normalisation of the energy operator $\varepsilon(x)$. When the energy perturbation is switched on, the spectrum in Fig. (7.d) undergoes continous deformations which can be followed by the truncation method. In particular, for $|\tau| \sim\left|T-T_{c}\right|$ sufficiently small and $h$ fixed, the variations in the energy levels are linear in $\tau$ and their direct measurement with the truncation method is given by

$$
\begin{aligned}
& \frac{\delta \mathcal{E}_{v a c}}{\delta m_{1}} \simeq-0.05 m_{1}^{0}, \\
& \frac{\delta m_{2}}{\delta m_{1}} \simeq 0.87, \\
& \frac{\delta m_{3}}{\delta m_{1}} \simeq 1.50 .
\end{aligned}
$$

The theoretical predictions for the same quantities are obtained plugging the values (5.7) into the first order formulae (3.17) and (3.16)

$$
\frac{\delta \mathcal{E}_{v a c}}{\delta m_{1}}=\frac{\langle 0|\varepsilon| 0\rangle}{F_{11}^{\varepsilon}(i \pi)} m_{1}^{0}=-0.0558 . . m_{1}^{0},
$$




$$
\begin{aligned}
& \frac{\delta m_{2}}{\delta m_{1}}=\frac{F_{22}^{\varepsilon}(i \pi)}{F_{11}^{\varepsilon}(i \pi)} \frac{m_{1}^{0}}{m_{2}^{0}}=0.8616 . . \\
& \frac{\delta m_{3}}{\delta m_{1}}=\frac{F_{33}^{\varepsilon}(i \pi)}{F_{11}^{\varepsilon}(i \pi)} \frac{m_{1}^{0}}{m_{3}^{0}}=1.5082 . .
\end{aligned}
$$

The agreement between the theoretical and numerical estimates should be regarded as quite satisfactory.

More dramatic is the effect of the perturbation on the five particles above the threshold. We have already mentioned that their stability at $\tau=0$ must be considered a consequence of the integrability of the theory which prevents any kind of inelastic process. They are then expected to decay in the perturbed, non-integrable theory. This is easily seen to be the case by analysing the effect of the perturbation on the finite size spectrum of the model. Indeed, since integrability is lost under the perturbation, level crossing in the spectrum is no longer allowed at $\tau \neq 0$. This means that the energy levels are now forced to repel each other at the former crossing points. Since each line associated to a particle above threshold crossed an infinite number of momentum lines at $\tau=0$ (Fig. (8.a)), it immediately becomes a "broken line" (almost) parallel to the threshold when the perturbation is switched on (Fig. (8.b)). This is exactly the signature of unstable particles in the finite volume; more precisely, the difference in the slopes of the broken line and the threshold is proportional to the width of the resonance [34].

In perturbation theory, the decay of the particles above threshold manifests itself through the appearence of a negative imaginary part in the mass. This is an effect which occurs though at the second order in $\tau$. To be specific, consider the lightest unstable particle $A_{4}$. It follows from (3.16) and (5.7) that the first order correction is real and is given by

$$
\frac{\delta m_{4}}{\delta m_{1}}=\frac{F_{44}^{\varepsilon}(i \pi)}{F_{11}^{\varepsilon}(i \pi)} \frac{m_{1}^{0}}{m_{4}^{0}}=1.1460 . .
$$

The computation of the second order correction requires the sum over a complete set of intermediate $n$-particle states and involves an energy denominator. Expressing the latter in the usual form

$$
\frac{1}{E-E_{n}+i \varepsilon}=\mathrm{P}\left(\frac{1}{E-E_{n}}\right)-i \pi \delta\left(E-E_{n}\right)
$$

one immediately concludes that the principal part gives rise to the second order correction 
to the real part of the mass while the delta function originates an imaginary part receiving a contribution only from the intermediate states whose total energy-momentum equals that of the external particle. For the particle $A_{4}$ we have

$$
\mathrm{I} m m_{4}^{2} \simeq-\frac{\tau^{2}}{2 m_{1}^{0} m_{4}^{0} \sinh \theta^{*}}\left|{ }_{0}\left\langle A_{4}(0)|\varepsilon(0)| A_{1}\left(\theta^{*}\right) A_{1}\left(-\theta^{*}\right)\right\rangle_{0}\right|^{2}
$$

where $\theta^{*}=\left|\operatorname{arccosh} \frac{m_{4}^{0}}{2 m_{1}^{0}}\right|$.

\section{Conclusions}

In this paper, we have shown that once the complete dynamics of two-dimensional exactly solvable models is known, this can be also extremely useful for investigating the structure of the quantum field theories close to the integrable directions. In particular, we have derived a perturbation theory for the non-integrable QFT based on the Form Factors of the exactly solvable relativistic models and we have considered the lowest term of the series in order to study the effects of a small perturbation which breaks the integrability of the unperturbed theory. We have discussed the variation of the mass spectrum, the shift in the vacuum energy density and the correction to the elastic part of the $S$-matrix, and we have successfully checked them against their numerical estimates obtained by the truncation method in two models with an underlying CFT, namely the minimal model $M_{(2,7)}$ and the Ising model.

It is worth pointing out that, specialising the perturbative formulas to the trivial case of a parallel perturbation along the original integrable model, one may obtain as a byproduct an infinite set of useful identities for the Form Factors of the stress-energy tensor of the original integrable field theory. While this appears to be as a convenient method of deriving them, at the same time it explains the reason of their validity because they can be regarded as consistency equations for the perturbative scheme built up on the Form Factors. Known features of the Form Factors of the integrable relativistic models are then deeply inter-related with the general structure of quantum field theories.

The methods illustrated in this paper may apply of course to other interesting physical situations in addition to those considered here. We would like to mention, for instance, 
two non-integrable lagrangian field theories which would be interesting to analyse in terms of their Form Factor perturbative series. The first one is given by

$$
L=\frac{1}{2}\left(\partial_{\mu} \varphi\right)^{2}-\frac{m^{2}}{6 g^{2}}\left[\left(1-\frac{\lambda}{3}\right)\left(2 e^{g \varphi}+e^{-2 g \varphi}\right)+\lambda\left(e^{g \varphi}+e^{-g \varphi}\right)\right]
$$

By varying the parameter $\lambda$ in the interval $[0,3]$, we can interpolate between the BulloghDodd and the Sinh-Gordon models. Both these field theories are separately integrable and the matrix elements of their local operators have been computed in [17, 18]. Notice that the $Z_{2}$ symmetry of the Sinh-Gordon model is always broken along the entire interpolating trajectory and it is only recovered at the end point $\lambda=3$.

The second non-integrable lagrangian model is obtained by adding higher "harmonics" to the original Sinh-Gordon interaction, the simplest example of this class of nonintegrable models being the so-called double Sinh-Gordon model

$$
L=\frac{1}{2}\left(\partial_{\mu} \varphi\right)^{2}-\frac{m^{2}}{2 g^{2}}[(1-4 \lambda) \cosh g \varphi+\lambda \cosh 2 g \varphi] .
$$

By varying $\lambda$ in the interval $\left[0, \frac{1}{4}\right]$, we can interpolate in this case between the integrable field theories given by the Sinh-Gordon theory with coupling constant $g$ and $2 g$.

Notice that for both examples $(\sqrt[6.1]{6})$ and $(6.2)$, the integrability of the two theories at the two extremes of the interpolation interval implies strong constraints for the ordinary perturbation theory based on the coupling constant $g$. It would be then interesting to learn more about those models by comparing the results obtained in ordinary Feynman diagram perturbation theory with those derived by the perturbative series based on the Form Factors.

Acknowledgements. We would like to thank J.L. Cardy and A. Schwimmer for useful discussions and suggestions. G.D. and P.S. were supported by EPSRC GR/J78044 and HEFCW grants, respectively. 


\section{Appendix A}

A simple example of quantum mechanics will help to clarify the simultaneous relevance of all the inelastic thresholds in any energy interval. Consider a one-dimensional inelastic scattering process of a particle of mass $m$ which hits a target with $n+1$ internal states of increasing energies $E_{i}(i=0,1, \ldots, n)$, where the excited states $\mid i>(i=1, \ldots, n)$ are given by $\left|i>=a_{i}^{\dagger}\right| 0>\left(a_{i}^{\dagger}\right.$ and $a_{i}$ denote the creation and annihilation operators, with standard commutation relations). The states of this system (target plus particle) are described by the wave function $\left|\Psi>=\sum_{i=0}^{n} a_{i}^{\dagger}\right| 0>\psi_{i}(x)$ and as Hamiltonian of the system we choose

$$
H=\frac{p^{2}}{2 m}+\sum_{i=0}^{n} E_{i} a_{i}^{\dagger} a_{i}-\frac{h^{2}}{2 m} \delta(x)\left[U_{0} \sum_{i=0}^{n} a_{i}^{\dagger} a_{i}+\sum_{i=1}^{n} U_{i}\left(a_{0}^{\dagger} a_{i}+a_{i}^{\dagger} a_{0}\right)\right]
$$

$U_{0}$ rules the elastic transition amplitudes $|i>\rightarrow| i>$ whereas $U_{i}$ are related to the inelastic reactions $|0>\leftrightarrow| i>$. For this toy-model, it is quite simple to determine the phase-shift $\delta_{0}$ and therefore the $S$-matrix element $S=e^{2 i \delta_{0}}$ relative to the elastic channel $|0>\rightarrow| 0>$. This is given by the formula

$$
\tan \delta_{0}=\frac{U_{0}}{2 k}-\sum_{l=1}^{n} \frac{U_{l}^{2}}{2 k\left(U_{0}+2 i k_{l}\right)},
$$

where

$$
\begin{aligned}
k^{2} & =\frac{2 m}{h}\left(E-E_{0}\right), \\
k_{j}^{2} & =\frac{2 m}{h}\left(E-E_{j}\right) .
\end{aligned}
$$

The phase shift $\delta_{0}$ is real when the energy $E$ is below the first threshold $E_{1}$ and complex above but it is worth to note that also in the elastic region $0<E<E_{1}$, its value is determined by all the inelastic parameters of the problems, i.e. $U_{l}$ and $k_{l}$.

\section{Appendix B}

As it is well known, a very effective algebraic description of integrable theories can be obtained in terms of the Faddeev-Zamolodchikov (FZ) creation and annihilation operators, 
$Z_{a}^{+}(\theta)$ and $Z_{a}(\theta)$. They satisfy the algebra

$$
\begin{aligned}
& Z_{a}^{+}\left(\theta_{1}\right) Z_{b}^{+}\left(\theta_{2}\right)=S_{a b}^{c d}\left(\theta_{1}-\theta_{2}\right) Z_{d}^{+}\left(\theta_{2}\right) Z_{c}^{+}\left(\theta_{1}\right) \\
& Z_{a}\left(\theta_{1}\right) Z_{b}\left(\theta_{2}\right)=S_{c d}^{a b}\left(\theta_{1}-\theta_{2}\right) Z_{d}\left(\theta_{2}\right) Z_{c}\left(\theta_{1}\right) \\
& Z_{a}\left(\theta_{1}\right) Z_{b}^{+}\left(\theta_{2}\right)=S_{b c}^{d a}\left(\theta_{2}-\theta_{1}\right) Z_{d}^{+}\left(\theta_{2}\right) Z_{c}\left(\theta_{1}\right)+2 \pi \delta_{a b} \delta\left(\theta_{1}-\theta_{2}\right)
\end{aligned}
$$

which can be regarded as a generalisation of the canonical commutation relations. The operator $Z_{a}(\theta)$ annihilates the vacuum while $Z_{a}^{+}(\theta)$ creates a particle of tipe $a$ with rapidity $\theta$. The space of states is generated by

$$
\begin{aligned}
& \left|a_{1}\left(\theta_{1}\right) \ldots a_{n}\left(\theta_{n}\right)\right\rangle=Z_{a_{1}}^{+}\left(\theta_{1}\right) \ldots Z_{a_{n}}^{+}\left(\theta_{n}\right)|0\rangle \\
& \left\langle a_{n}\left(\theta_{n}\right) \ldots a_{1}\left(\theta_{1}\right)\right|=\langle 0| Z_{a_{n}}\left(\theta_{n}\right) \ldots Z_{a_{1}}\left(\theta_{1}\right) .
\end{aligned}
$$

The physical asymptotic states can be selected through the following ordering prescription over rapidities: the states (B.2) are "In" states if $\theta_{1}>\theta_{2}>\ldots>\theta_{n}$, and "Out" states if $\theta_{1}<\theta_{2}<\ldots<\theta_{n}$.

It is easily checked that the unitarity and Yang-Baxter equations for the S-matrix can be obtained requiring respectively the consistency under double application and the associativity of the FZ algebra (B.1). Concerning the remaining fundamental property of the S-matrix, namely the crossing relation

$$
S_{a b}^{c d}(\theta)=S_{\overline{d a}}^{\bar{b} c}(i \pi-\theta)
$$

let's formally perform in the last equation in (B.1) the analytic continuation $\theta_{2} \longrightarrow \theta_{2}+i \pi$. Then, substituting particles $b$ and $d$ with their antiparticles $\bar{b}$ and $\bar{d}$ and using eq. (B.3), we obtain

$$
Z_{a}\left(\theta_{1}\right) Z_{\bar{b}}^{+}\left(\theta_{2}+i \pi\right)=S_{c d}^{a b}(\theta) Z_{\bar{d}}^{+}\left(\theta_{2}+i \pi\right) Z_{c}\left(\theta_{1}\right), \quad\left(\theta_{1} \neq \theta_{2}\right)
$$

Comparison with the second equation in (B.1) suggests the identification

$$
Z_{\bar{b}}^{+}(\theta+i \pi)=-Z_{b}(\theta)
$$

The proportionality constant -1 has been chosen in order to fit the canonical cases corresponding to $S_{a b}^{c d}(\theta)= \pm 1$. Indeed we recall that the Fourier decomposition of a free 
(e.g. bosonic) field

$$
\varphi(x)=\frac{1}{2 \pi} \int \frac{d k^{1}}{k^{0}}\left[a(k) e^{-i k x}+a^{+}(k) e^{i k x}\right],
$$

immediately leads to the representation

$$
\begin{aligned}
& a(k)=-\frac{i}{2} \int d x^{1} e^{i k x} \stackrel{\leftrightarrow}{\partial}_{0} \varphi(x)=f(k), \\
& a^{+}(k)=\frac{i}{2} \int d x^{1} e^{-i k x} \stackrel{\leftrightarrow}{\partial}_{0} \varphi(x)=-f(-k)
\end{aligned}
$$

Consider the two matrix elements

$$
\begin{gathered}
{ }_{c_{0}} F_{a_{1} \ldots a_{n}}^{\mathcal{O}}\left(\theta \mid \theta_{1}, \ldots, \theta_{n}\right)=\left\langle 0\left|\left[Z_{c_{0}}(\theta), \mathcal{O}(0)\right]\right| a_{1}\left(\theta_{1}\right), \ldots, a_{n}\left(\theta_{n}\right)\right\rangle+\left\langle 0\left|\mathcal{O}(0) Z_{c_{0}}(\theta)\right| a_{1}\left(\theta_{1}\right), \ldots, a_{n}\left(\theta_{n}\right)\right\rangle, \\
F_{\bar{c}_{0} a_{1} \ldots a_{n}}^{\mathcal{O}}\left(\theta, \theta_{1}, \ldots, \theta_{n}\right)=\left\langle 0\left|\left[\mathcal{O}(0), Z_{\bar{c}_{0}}^{+}\right]\right| a_{1}\left(\theta_{1}\right), \ldots, a_{n}\left(\theta_{n}\right)\right\rangle
\end{gathered}
$$

We can use the identification (B.5) and the FZ algebra to recognize that the first matrix element can be written as

$$
\begin{aligned}
& c_{0} F_{a_{1} \ldots a_{n}}^{\mathcal{O}}\left(\theta \mid \theta_{1}, \ldots, \theta_{n}\right)=F_{\bar{c}_{0} a_{1} \ldots a_{n}}^{\mathcal{O}}\left(\theta+i \pi, \theta_{1}, \ldots, \theta_{n}\right)+ \\
& +2 \pi \sum_{i=1}^{n} \delta_{c_{i-1} a_{i}} \delta\left(\theta-\theta_{i}\right)\left[\prod_{k=1}^{i-1} S_{a_{k} c_{k}}^{d_{k} c_{k-1}}\left(\theta_{k}-\theta\right)\right] F_{d_{1} \ldots d_{i-1} a_{i+1} \ldots a_{n}}^{\mathcal{O}}\left(\theta_{1}, \ldots \theta_{i-1}, \theta_{i+1}, \ldots, \theta_{n}\right) .
\end{aligned}
$$

This result is represented pictorially in fig.A1. As an illustration of this crossing procedure we write down esplicitely the two matrix elements involved in the evaluation of the mass and amplitude variations (3.16) and (3.21)

$$
\begin{gathered}
{ }_{b} F_{a}^{\mathcal{O}}\left(\theta_{2} \mid \theta_{1}\right)=F_{\bar{b} a}^{\mathcal{O}}\left(\theta_{2}+i \pi, \theta_{1}\right)+2 \pi \delta_{a b} \delta\left(\theta_{1}-\theta_{2}\right)\langle 0|\mathcal{O}| 0\rangle \\
{ }_{c d} F_{a b}^{\mathcal{O}}\left(\theta_{3}, \theta_{4} \mid \theta_{1}, \theta_{2}\right)=F_{\bar{c} \bar{d} a b}^{\mathcal{O}}\left(\theta_{3}+i \pi, \theta_{4}+i \pi, \theta_{1}, \theta_{2}\right)+ \\
2 \pi \delta\left(\theta_{1}-\theta_{3}\right) S_{\bar{d} a}^{e c}\left(i \pi+\theta_{4}-\theta_{3}\right) F_{e b}^{\mathcal{O}}\left(\theta_{4}+i \pi, \theta_{2}\right)+ \\
+2 \pi \delta\left(\theta_{2}-\theta_{4}\right) S_{a b}^{e d}\left(\theta_{1}-\theta_{2}\right) F_{\bar{c} e}^{\mathcal{O}}\left(\theta_{3}+i \pi, \theta_{1}\right)+ \\
+(2 \pi)^{2} \delta\left(\theta_{2}-\theta_{4}\right) \delta\left(\theta_{1}-\theta_{3}\right) S_{a b}^{c d}\left(\theta_{1}-\theta_{2}\right)\langle 0|\mathcal{O}| 0\rangle
\end{gathered}
$$

In the last equation we excluded the "resonances" $\theta_{4}=\theta_{1}$ and $\theta_{3}=\theta_{2}$. 


\section{Appendix $\mathrm{C}$}

In this section, we collect the needed $\mathrm{FF}$ of the operator $\Phi_{1,2}$ relative to the $\Phi_{1,3}$ deformation of the minimal non-unitarity model $M_{(2,7)}$. They are given by

$$
\begin{gathered}
\left\langle 0\left|\Phi_{1,2}\right| 0\right\rangle=\frac{c_{0}}{2 \cos \frac{2 \pi}{5}}, \\
F_{11}(\theta)=-c_{0} 4 \sin \frac{\pi}{5} \frac{F_{11}^{\min }(\theta)}{\mathcal{P}_{\frac{2}{5}}(\theta)} \\
F_{22}(\theta)=-c_{0} 8 \sin \frac{2 \pi}{5} \frac{\left(\cosh \theta+4 \cos \frac{\pi}{5} \sin ^{2} \frac{2 \pi}{5}\right) F_{22}^{\min }(\theta)}{\mathcal{P}_{\frac{2}{5}}(\theta) \mathcal{P}_{\frac{3}{5}}(\theta) \mathcal{P}_{\frac{4}{5}}(\theta)}
\end{gathered}
$$

and

$$
F_{1111}\left(\theta_{i j}\right)=c_{0} \frac{\sin ^{4} \frac{\pi}{5}}{\cos ^{7} \frac{\pi}{5} G_{\frac{2}{5}}^{2}(0)} \mathcal{Q}\left(\theta_{i j}\right) \prod_{l<k}^{4} \frac{F_{11}^{\min }\left(\theta_{l k}\right)}{\cosh \frac{\theta_{l k}}{2} \mathcal{P}_{\frac{2}{5}}\left(\theta_{l k}\right)} .
$$

In the above formulas, the functions

$$
F_{11}^{\min }(\theta)=-i \sinh \frac{\theta}{2} G_{\frac{2}{5}}(\theta)
$$

and

$$
F_{22}^{\min }(\theta)=-i \sinh \frac{\theta}{2} G_{\frac{2}{5}}(\theta) G_{\frac{3}{5}}(\theta) G_{\frac{4}{5}}(\theta)
$$

where

$$
G_{\alpha}(\theta)=\exp \left[2 \int_{0}^{+\infty} \frac{d t}{t} \frac{\cosh \left(\alpha-\frac{1}{2}\right) t}{\cosh \frac{t}{2} \sinh t} \sin ^{2} \frac{(i \pi-\theta) t}{2 \pi}\right],
$$

solve the monodromy problem for the form factors, while the functions

$$
\mathcal{P}_{\alpha}(\theta)=\frac{\cos \pi \alpha-\cosh \theta}{2 \cos ^{2} \frac{\pi \alpha}{2}}
$$

appearing in the denominators are in coincidence with the bound state structure of the theory. The function $\mathcal{Q}$ in the four particle form factor is defined as

$$
\begin{aligned}
& \mathcal{Q}\left(\theta_{i j}\right)= \\
& \left(\sum_{l<k}^{4} \cosh \theta_{l k}+4 \sin ^{2} \frac{2 \pi}{5}\right)\left[\cosh \frac{\theta_{12}}{2} \cosh \frac{\theta_{34}}{2}+\cosh \frac{\theta_{13}}{2} \cosh \frac{\theta_{24}}{2}+\cosh \frac{\theta_{14}}{2} \cosh \frac{\theta_{23}}{2}\right]+ \\
& \cos \frac{\pi}{5}\left[\cosh \frac{1}{2}\left(\theta_{12}+\theta_{13}+\theta_{14}\right)+\cosh \frac{1}{2}\left(\theta_{21}+\theta_{23}+\theta_{24}\right)+\right. \\
& \left.\cosh \frac{1}{2}\left(\theta_{31}+\theta_{32}+\theta_{34}\right)+\cosh \frac{1}{2}\left(\theta_{41}+\theta_{42}+\theta_{43}\right)\right] .
\end{aligned}
$$


Notice that the constant $c_{0}$ in front of all Form Factors depends on the (non-universal) normalisation $\square$ of the field $\Phi_{1,2}(x)$. We can get around the difficult problem of determining this constant by considering universal ratios.

17 The value of $c_{0}$ corresponding to the standard CFT normalisation, identified by the two-point function behaviour $<\Phi_{12}(x) \Phi_{12}(0)>\stackrel{x \rightarrow 0}{\longrightarrow} x^{-4 \Delta_{12}}$, can be hardly determined without resumming the entire spectral series of the two-point function. This turns out to be in general quite a difficult problem. 


\section{References}

[1] A.B. Zamolodchikov and Al.B. Zamolodchikov, Ann. Phys. 120 (1979) 253.

[2] A.B. Zamolodchikov, in Advanced Studies in Pure Mathematics 19 (1989) 641; Int. J. Mod. Phys. A3 (1988) 743.

[3] V.A. Fateev and A.B. Zamolodchikov, Int. Journ. of Mod. Phys. A 5 (1990), 1025; A.B. Zamolodchikov, Mod. Phys. Lett. A 6 (1991), 1807.

[4] G. Mussardo, Phys. Reports 218 (1992), 215 and references therein.

[5] N.Yu. Reshetikhin and F.A. Smirnov, Commun. Math. Phys. 131 (1990), 157; F.A. Smirnov, Int. J. Mod. Phys. A4 (1989), 4231; Nucl. Phys. B337 (1990), 156;

[6] F.A. Smirnov, Int. J. Mod. Phys. A6 (1991), 1407.

[7] P.G. Freund, T.R. Klassen and E. Melzer, Phys. Lett. B 229 (1989), 243.

[8] A. Koubek and G. Mussardo, Phys. Lett. B 266 (1991), 363; A. Koubek, M.J. Martins and G. Mussardo, Nucl. Phys. B 368 (1992), 591.

[9] A.E. Arinshtein, V.A. Fateev and A.B. Zamolodchikov, Phys. Lett. B 87 (1979), 389; H.W. Braden, E. Corrigan, P.E. Dorey and R. Sasaki, Nucl. Phys. B 338 (1990), 689; G. Sotkov and C.J. Zhu, Phys. Lett. B 229 (1989), 391; P. Christe and G. Mussardo, Nucl. Phys. B 330 (1990), 465.

[10] Al.B. Zamolodchikov, Nucl. Phys. B342 (1990), 695; V.A. Fateev, Phys. Lett. B 324 (1994), 45.

[11] G. Delfino and G. Mussardo, Nucl. Phys. B 455 (1995), 724.

[12] B. Berg, M. Karowsky and P. Weisz, Phys. Rev. D19 (1979) 2477; M. Karowsky and P. Weisz, Nucl. Phys. B139 (1978) 445; M. Karowsky, Phys. Rep. 49 (1979) 229.

[13] F.A. Smirnov, Form Factors in Completely Integrable Models of Quantum Field Theories (World Scientific) 1992, and references therein. 
[14] A.Koubek, Nucl. Phys. B 428 (1994), 655; Nucl. Phys. B 435 (1995), 703.

[15] V.P. Yurov and Al.B. Zamolodchikov, Int. J. Mod. Phys. A 6 (1991), 3419; J.L. Cardy and G. Mussardo, Nucl. Phys. B 340 (1990), 387; O. Babelon and D. Bernard, Phys. Lett. B 288 (1992), 113.

[16] Al.B. Zamolodchikov, Nucl. Phys. B 348 (1991), 619; G. Delfino and G. Mussardo, Phys. Lett. B 324 (1994), 40; J. Balog, Phys. Lett. B 300 (1993), 145; J.L. Cardy and G. Mussardo, Nucl. Phys. B 410 [FS] (1993), 451.

[17] A. Fring, G. Mussardo and P. Simonetti, Nucl. Phys. B 393 (1993), 413; A. Koubek and G. Mussardo, Phys. Lett. B 311 (1993), 193;

[18] G. Mussardo and P. Simonetti, Int. J. Mod. Phys. A 9 (1994), 3307; A. Fring, G. Mussardo and P. Simonetti, Phys. Lett. B 307 (1993), 413.

[19] G. Delfino, G. Mussardo and P. Simonetti, Phys. Rev. D 51 (1995), R6620.

[20] P. Christe, Int. J. Mod. Phys. A6 (1991), 5271; A. Koubek, Phys. Lett. B 346 (1995), 275; F.A. Smirnov, Nucl. Phys. B 453 (1995), 807; F.A. Smirnov, J. Phys. 18 (1984) L 873; J. Phys. 19 (1986), L 575; A.N. Kirillov and F.A. Smirnov, Phys. Lett. B198 (1987), 506; F.A. Smirnov, Comm. Math. Phys. bf 132 (1990), 415; S. Lukyanov and S.L. Shatasvili, Phys. Lett. B 298 (1993), 111; S. Lukyanov, Comm. Math. Phys. 167 (1995), 183; S. Lukyanov, Phys. Lett. B 235 (1994), 409.

[21] A. Messiah, Quantum Mechanics, North Holland Publishing Company, Amsterdam 1961.

[22] T.T. Wu, B.M. McCoy, C.A. Tracy and M. Barouch, Phys. Rev. B13 (1976), 316; B.M. McCoy, C. Tracy and T.T. Wu, Phys. Rev. Lett. 38 (1977), 793; B.M. McCoy and T.T. Wu, Phys. Rev. Lett. 45 (1980), 675; J. Palmer and C.A. Tracy, Adv. in Applied Math. 2 (1981) 329; J.H.H. Perk, Phys. Lett. 79A (1990), 1; B.M. McCoy, J.H.H. Perk and T.T. Wu, Phys. Rev. Lett. 46 (1981), 757; B.M. McCoy and C. Tracy, Phys Rev. B12 (1975) 368; M. M. McCoy, C.A. Tracy and T.T. Wu, Journ. Math. Phys. 18 (1977), 1058; 
[23] B.M. McCoy and T.T. Wu, The Two-Dimensional Ising Model (Harvard University Press, Cambridge, 1973).

[24] M. Sato, T. Miwa and M. Jimbo, Proc. Jap. Acad. 53A (1977) 147,153, 183.

[25] B.M. McCoy and T.T. Wu, Phys. Rev. D 18 (1978), 1259.

[26] B.M. McCoy, The connection between statistical mechanics and quantum field theory, ITPSB 94-07, hep-th/9403084.

[27] B.M. McCoy and W.P. Orrick, Analyticity and Integrability in the Chiral Potts Model, ITPSB 95-14, hep-th/9509004.

[28] A.A. Belavin, A.M. Polyakov and A.B. Zamolodchikov, Nucl. Phys. B 241 (1984), 333; D. Friedan, Z. Qiu and S. Shenker, Phys. Rev. Lett. 52 (1984), 1575; C. Itzykson, H. Saleur and J.B. Zuber, Conformal Invariance and Applications to Statistical Mechanics, (World Scientific, Singapore 1988) and references therein.

[29] J.L. Cardy, Nucl. Phys. B 270 (1986), 186; in Champs, cordes et phenomenes critiques, Proc. Les Houches Summer School, ed. E. Brezin and J. Zinn-Justin (NorthHolland, Amsterdam, 1989).

[30] G. Delfino, G. Mussardo and P. Simonetti, in preparation.

[31] G. Delfino and P. Simonetti, in preparation.

[32] V.V. Bazhanov, B. Nienhuis, and S.O. Warnaar, Phys. Lett. B 322 (1994), 198.

[33] P.G. Lauwers and V. Rittenberg, Phys. Lett. B 233 (1989), 197; P.G. Lauwers and V. Rittenberg, Numerical Estimates of the Spin-Spin Correlation Function of the Critical 2-D Ising Model in a Magnetic Field, Bonn-HE-89-11; C. Destri, F. Di Renzo, E. Onofri, P. Rossi and G.P. Tecchiolli, Phys. Lett. B 278 (1992), 311.

[34] M. Luscher and U. Wolff, Nucl. Phys. B 339 (1990), 222; M. Luscher, in Champs, cordes et phenomenes critiques, Proc. Les Houches Summer School, ed. E. Brezin and J. Zinn-Justin (North-Holland, Amsterdam, 1989). 
[35] V.P. Yurov and Al. B. Zamolodchikov, Int. J. Mod. Phys. A 5 (1990), 3221.

[36] M. Lassig and G. Mussardo, Computer Phys. Comm. 66 (1991), 71.

[37] G. von Gehlen, Nucl. Phys. B330 1990), 741; M. Henkel, Phys. Lett. B247 (1990), 567; M. Lässig, G. Mussardo and J.L. Cardy, Nucl. Phys. B348 (1991), 591; V.P. Yurov and Al.B. Zamolodchikov, Int. J. Mod. Phys. A 6 (1991), 4557; M. Henkel and H. Saleur, J. Phys. A 22 L513 (1989); I.R. Sagdeev and A.B. Zamolodchikov, Mod. Phys. Lett. B 3 (1989), 1375.

[38] M. Lassig and M.J. Martins, Nucl. Phys. B 354 (1991), 666.

[39] M.J. Martins, Int. J. Mod. Phys. A 7 (1992), 7753.

[40] B.M. McCoy and M.L. Yan, Nucl. Phys. B 257 [FS14] (1985), 303.

[41] Vl.S. Dotsenko, Nucl. Phys. B 314 (1989), 687; Int. J. Mod. Phys. B 4 (1990), 1039. 


\section{Figure Caption}

Figure 1. Analytic structure of two-body $S$-matrix in non-integrable field theories. The crosses indicate the location of the bound state poles whereas the thick lines the branch cuts originating at the thresholds.

Figure 2 . Renormalization Group trajectories associated to the field theories: integrable directions (continous lines) and non-integrable one (dashed line).

Figure 3 . First energy levels of the $\Phi_{1,3}$ integrable deformation of the conformal model $M_{(2,7)}$ as functions of the strip width $R$.

Figure 4 . Mass ratio of the non-integrable deformations of the conformal model $M_{(2,7)}$ as function of $\chi$.

Figure 5 . Phase space in the vicinity of the critical point of the 2-d Ising model.

Figure 6 . Analytic structure in momentum space of the spin-spin correlation function of the Ising model relative to the points $(a, \ldots, g)$ in Fig. 5.

Figure 7 . Numerical spectrum of the Ising model relative to the points $(a, \ldots, g)$ in Figure 5.

Figure 8 . (a) The energy level corresponding to a stable particle above threshold crosses several momentum lines in the integrable theory; (b) removal of the crossing points implying the decay of the particle above theshold in the perturbed, non-integrable theory.

Figure 9 . Crossing relation for the Form Factors. 


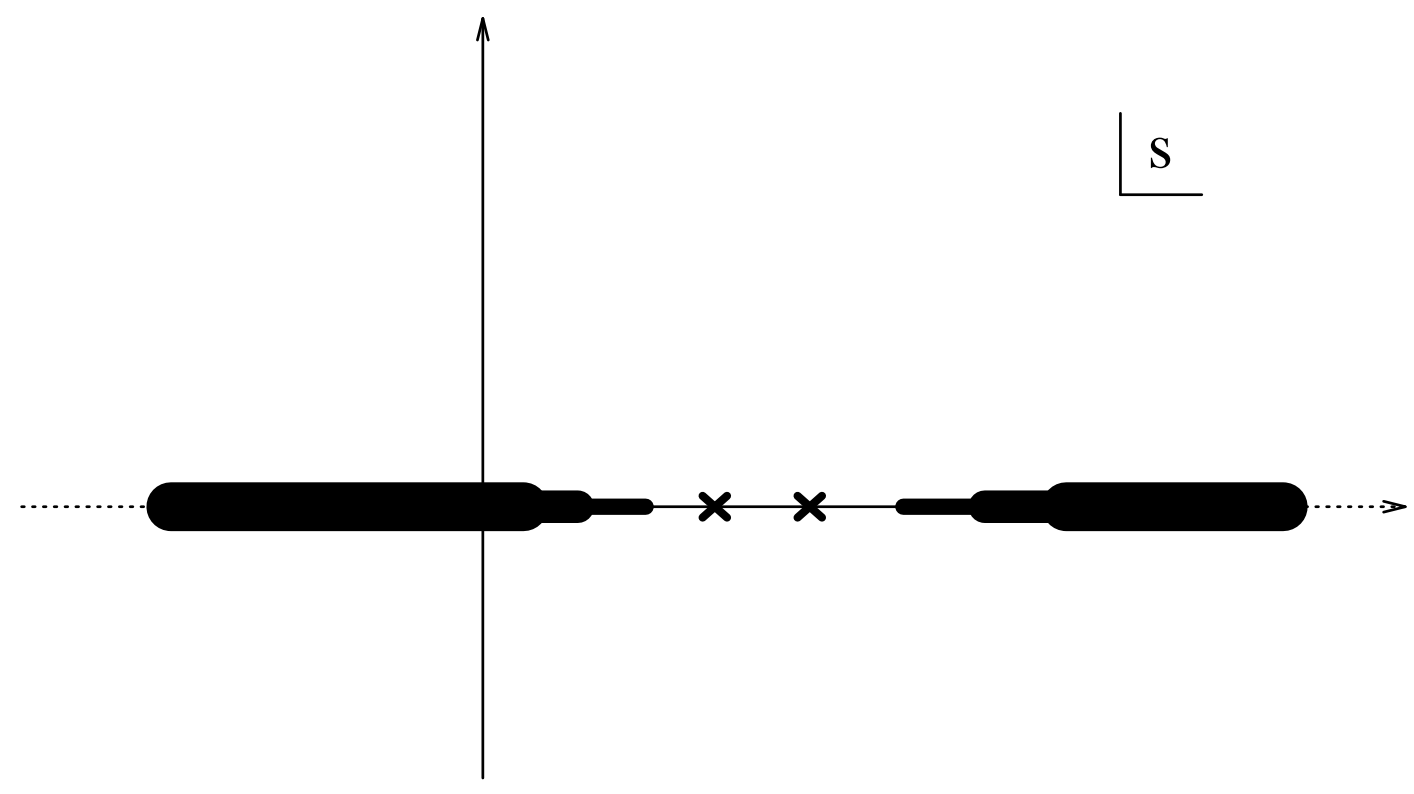

Figure 1

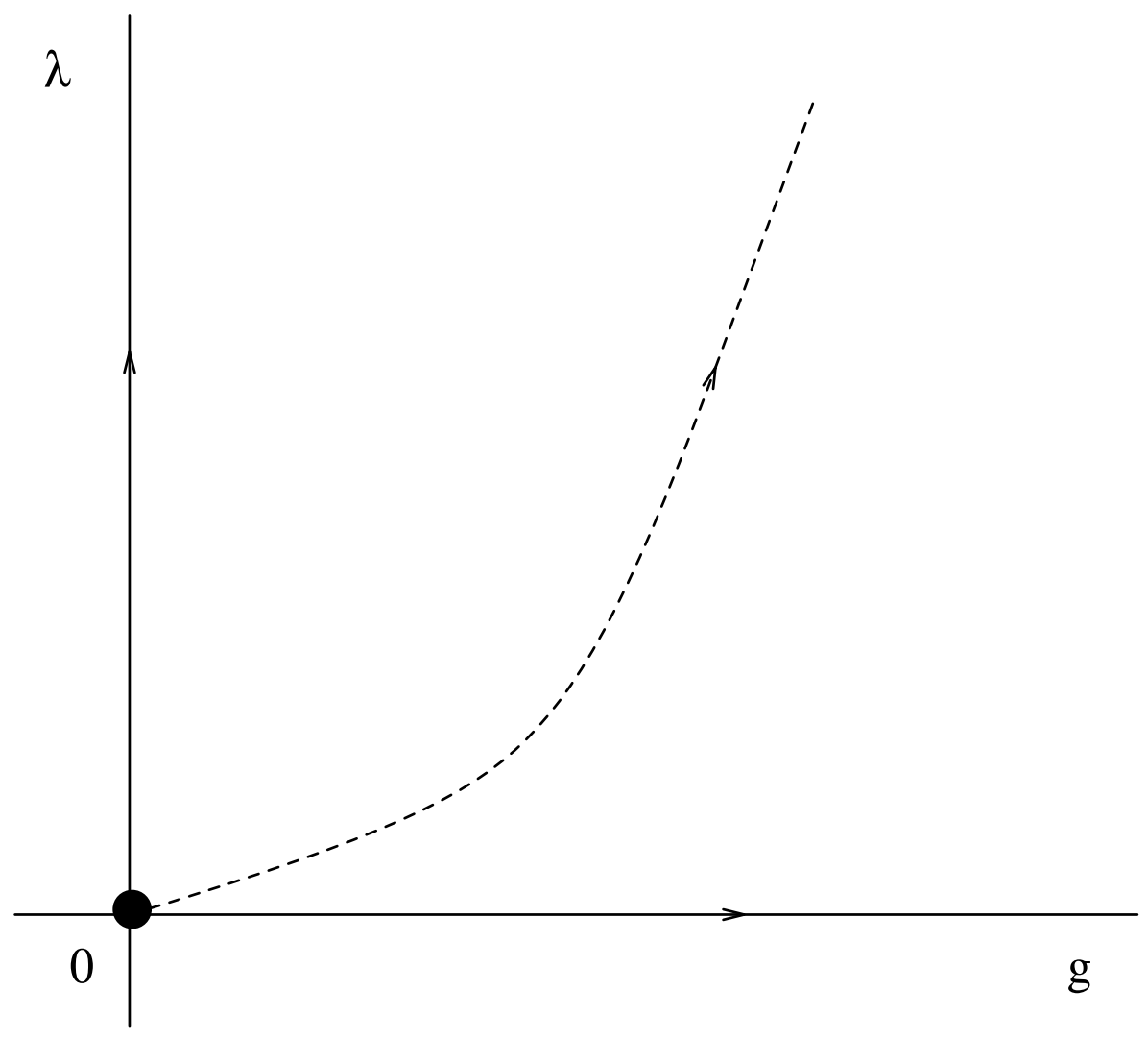

Figure 2 


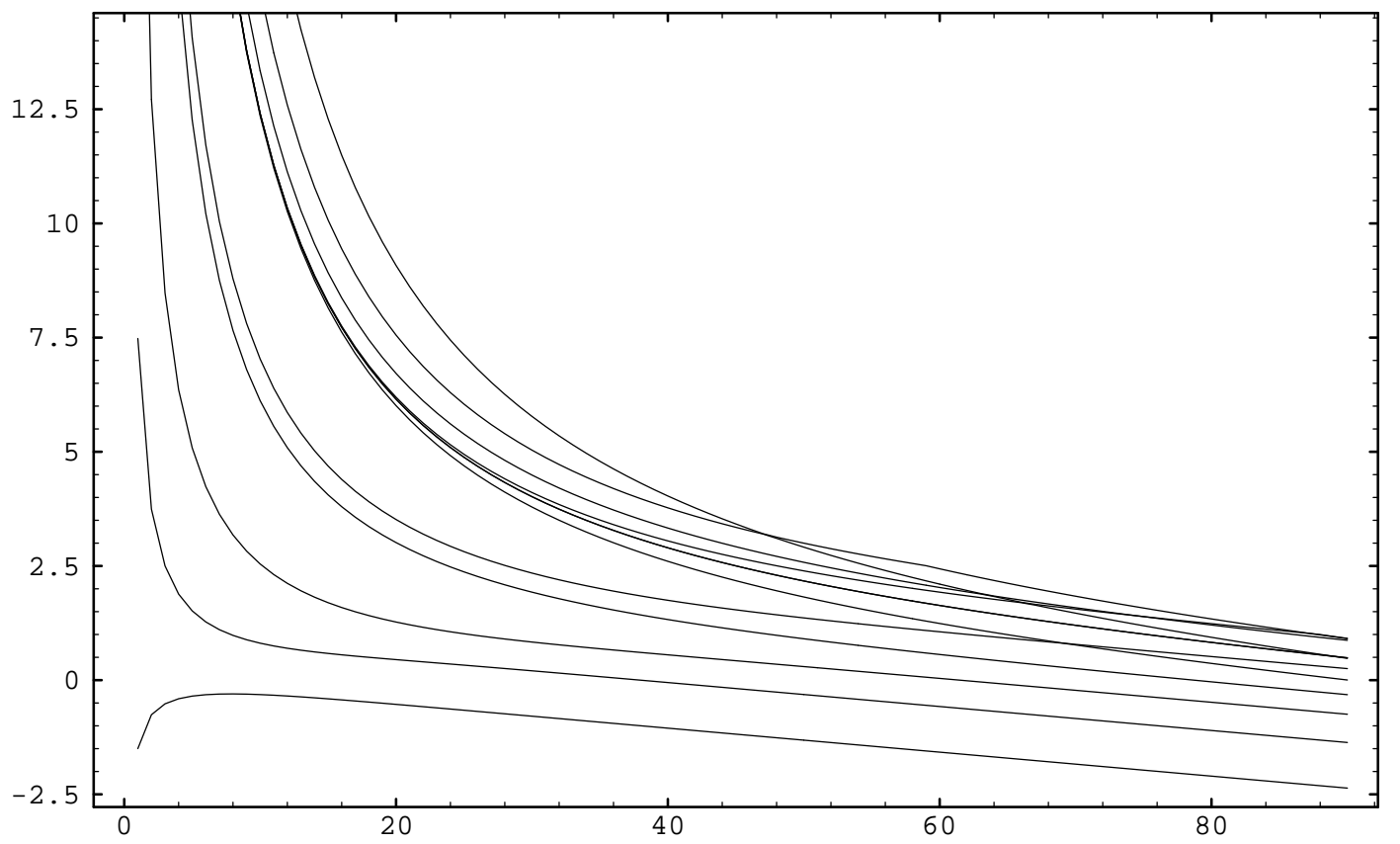

Figure 3 


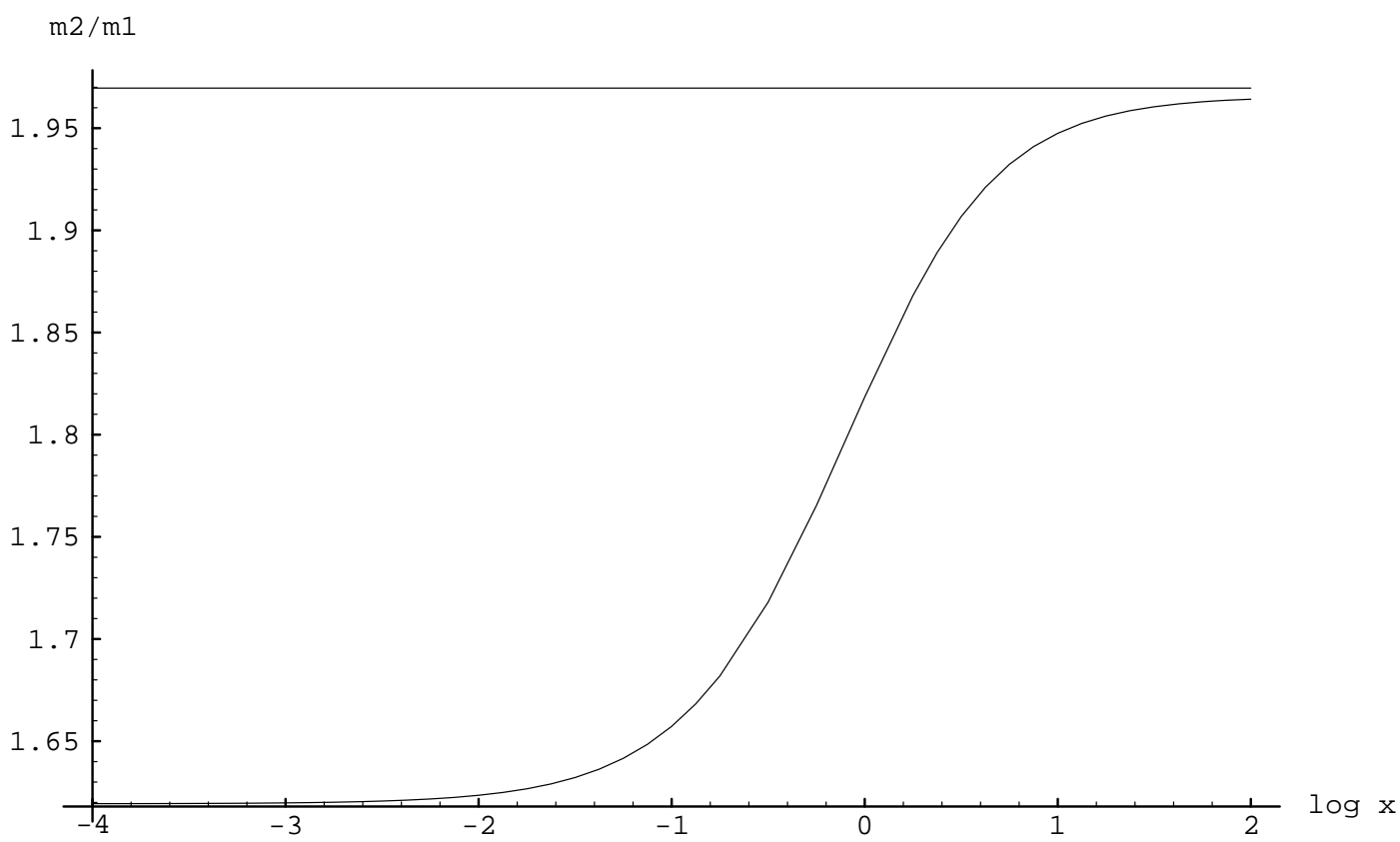

Figure 4 


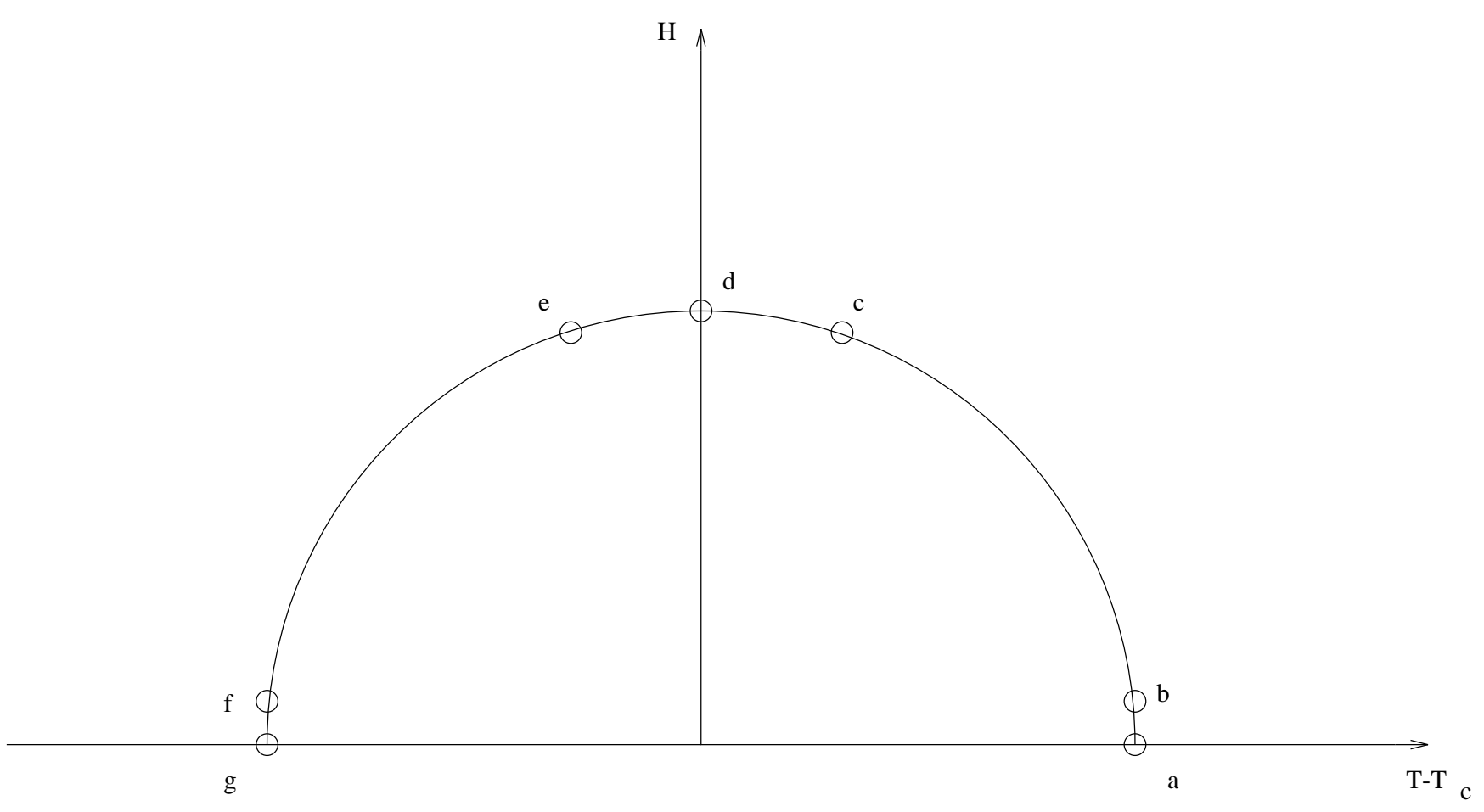

Figure 5 

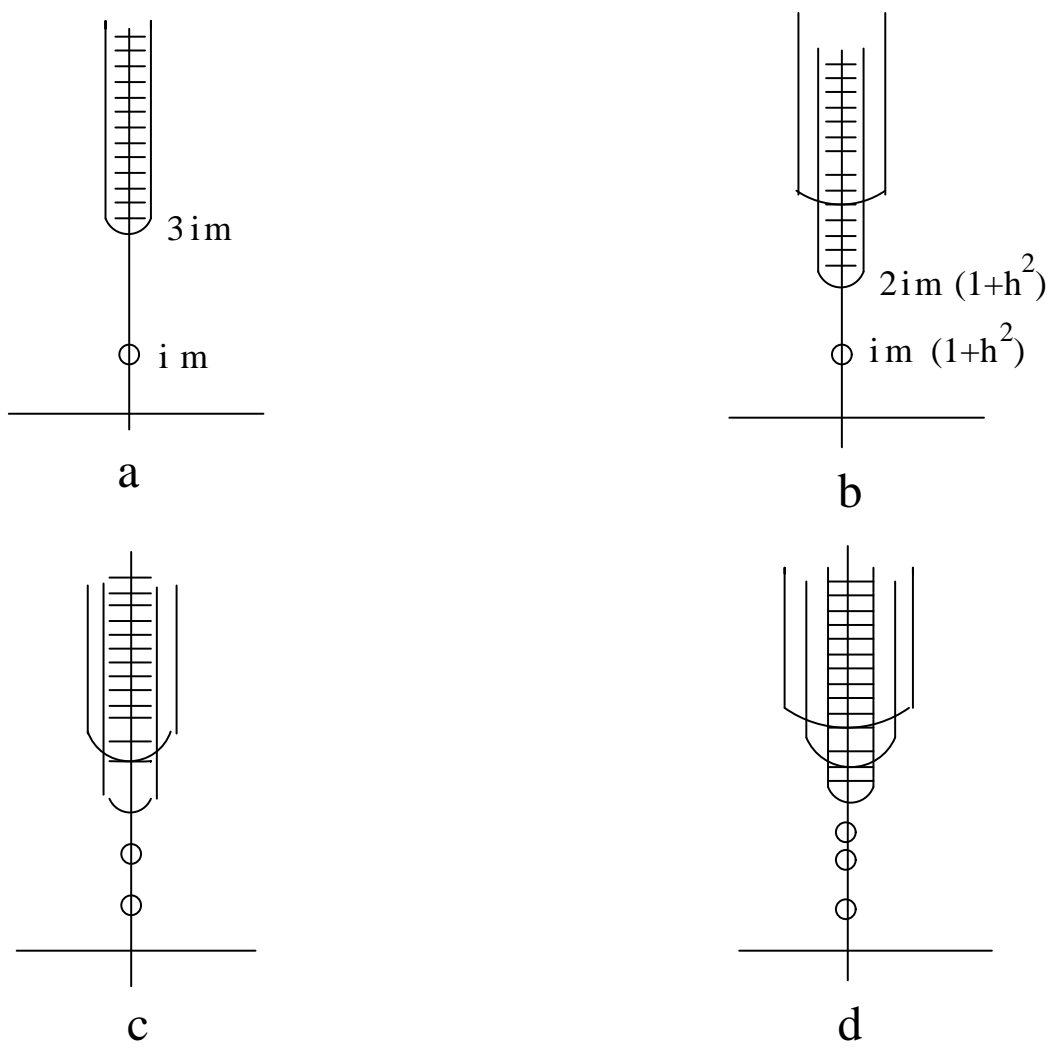

Figure 6 

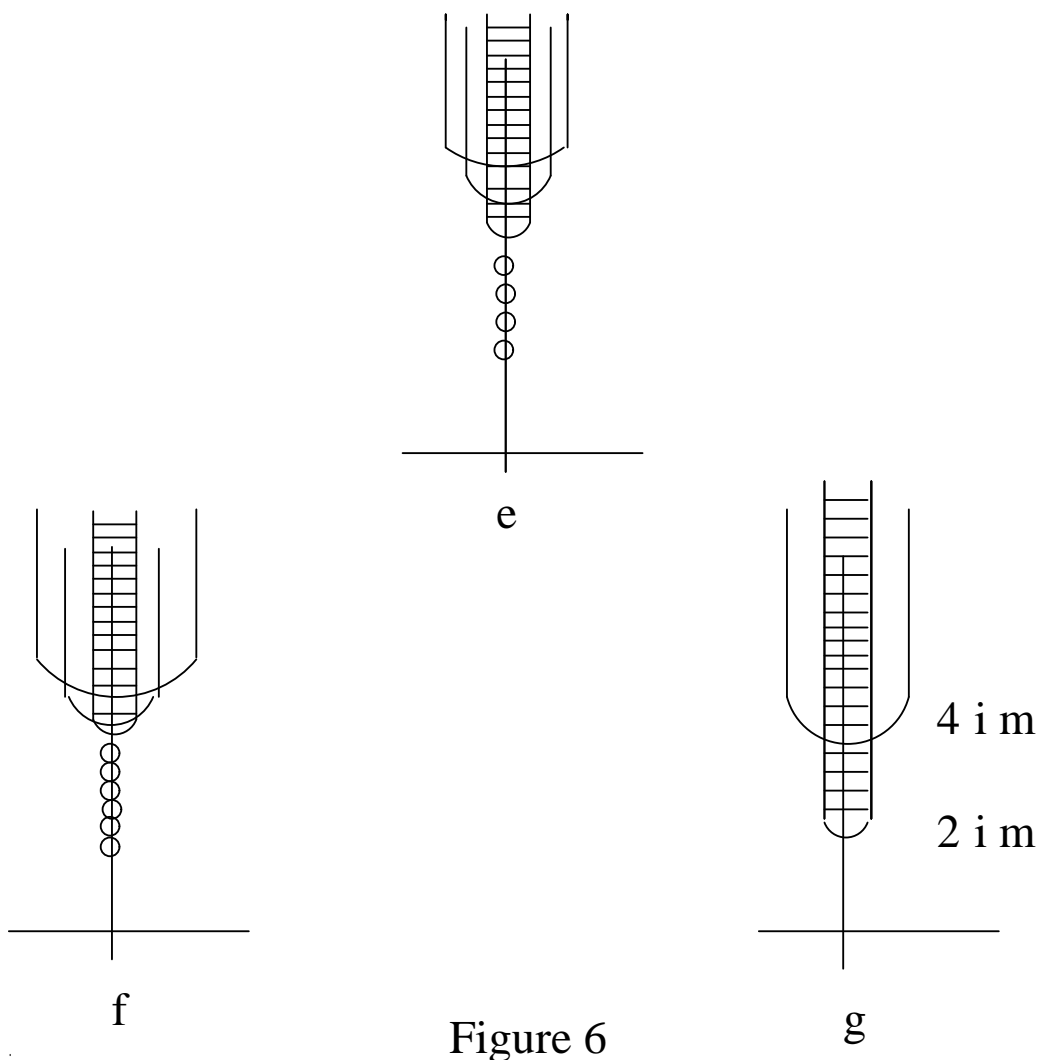

Figure 6 


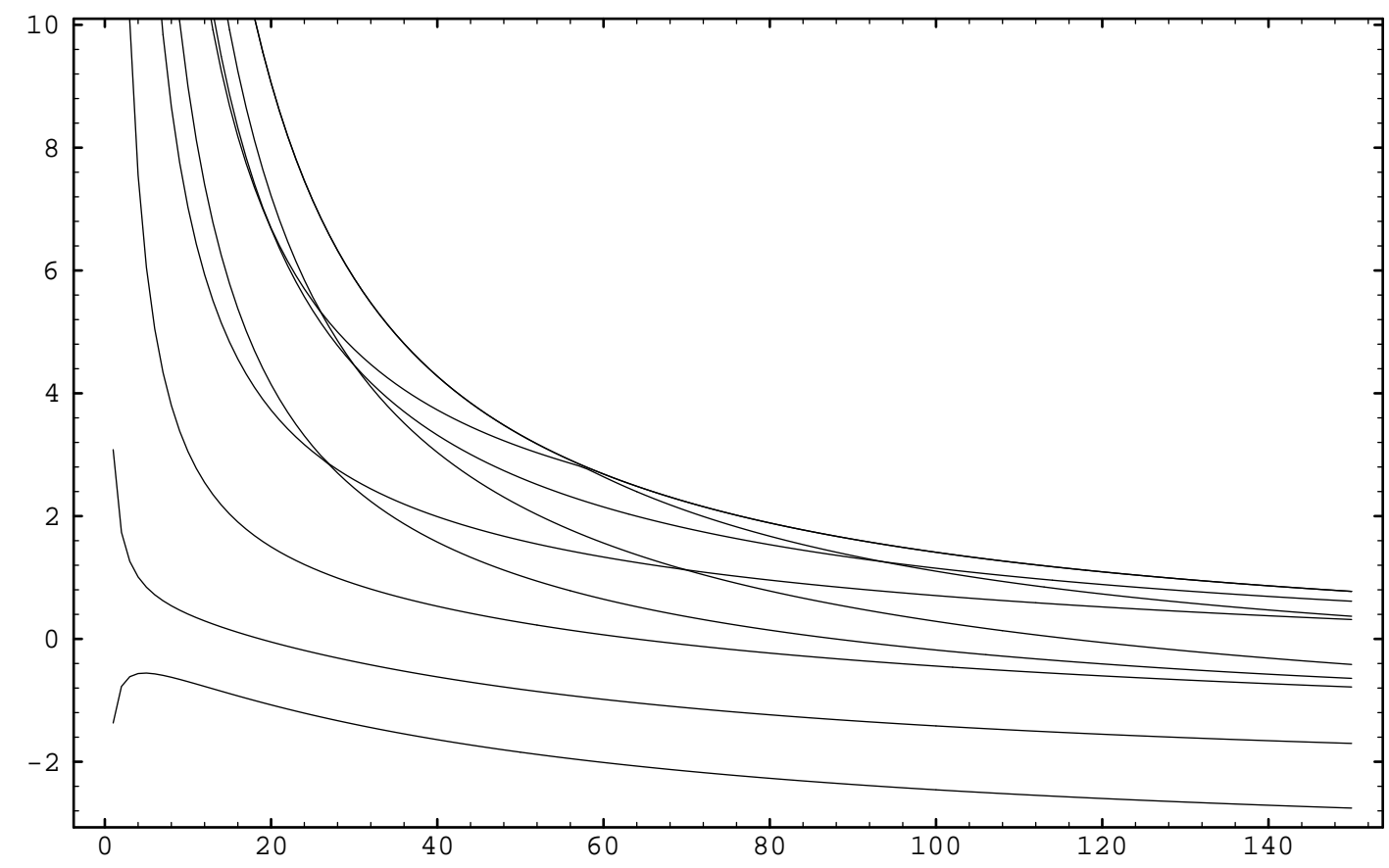

Figure 7.a 


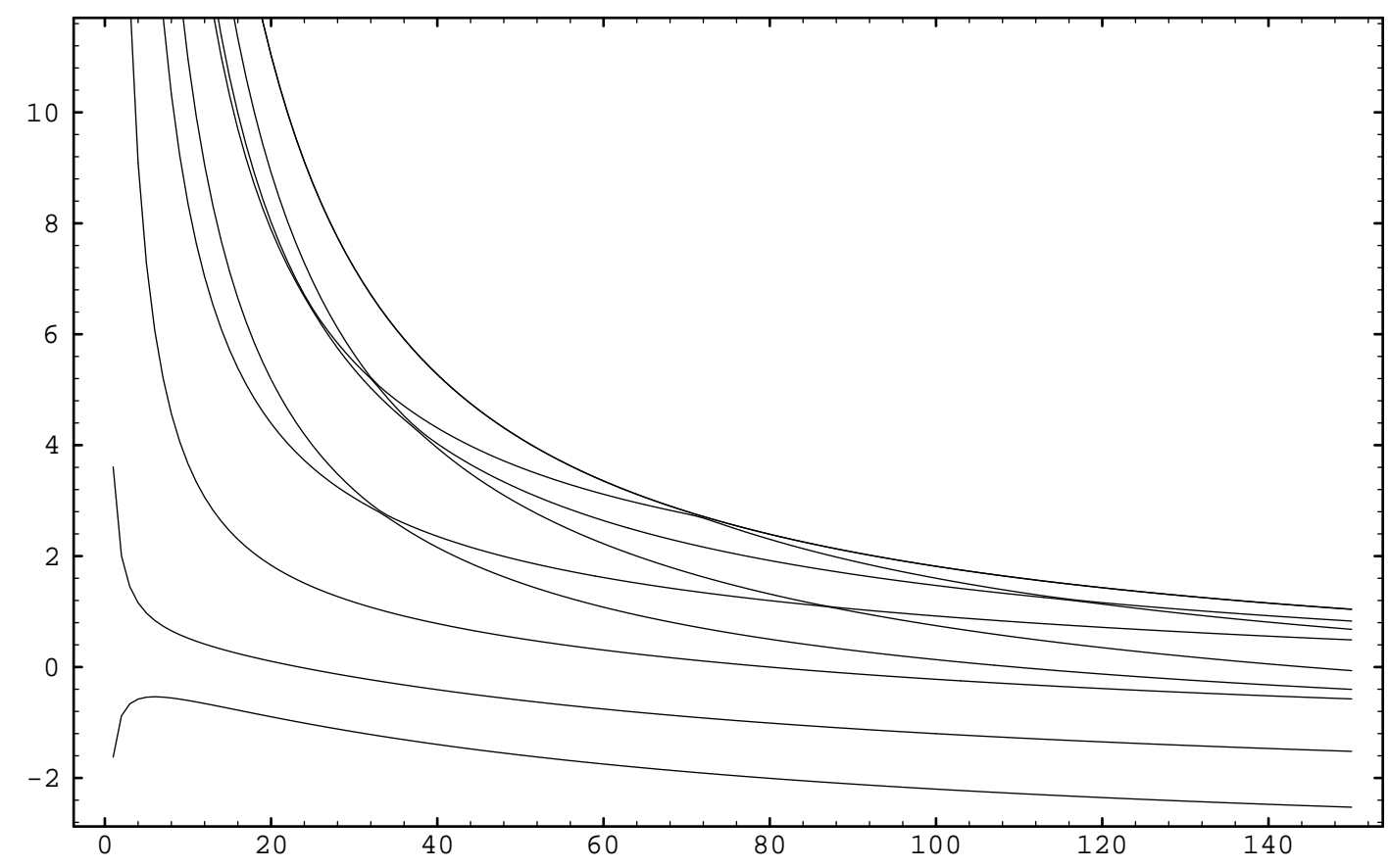

Figure 7.b 


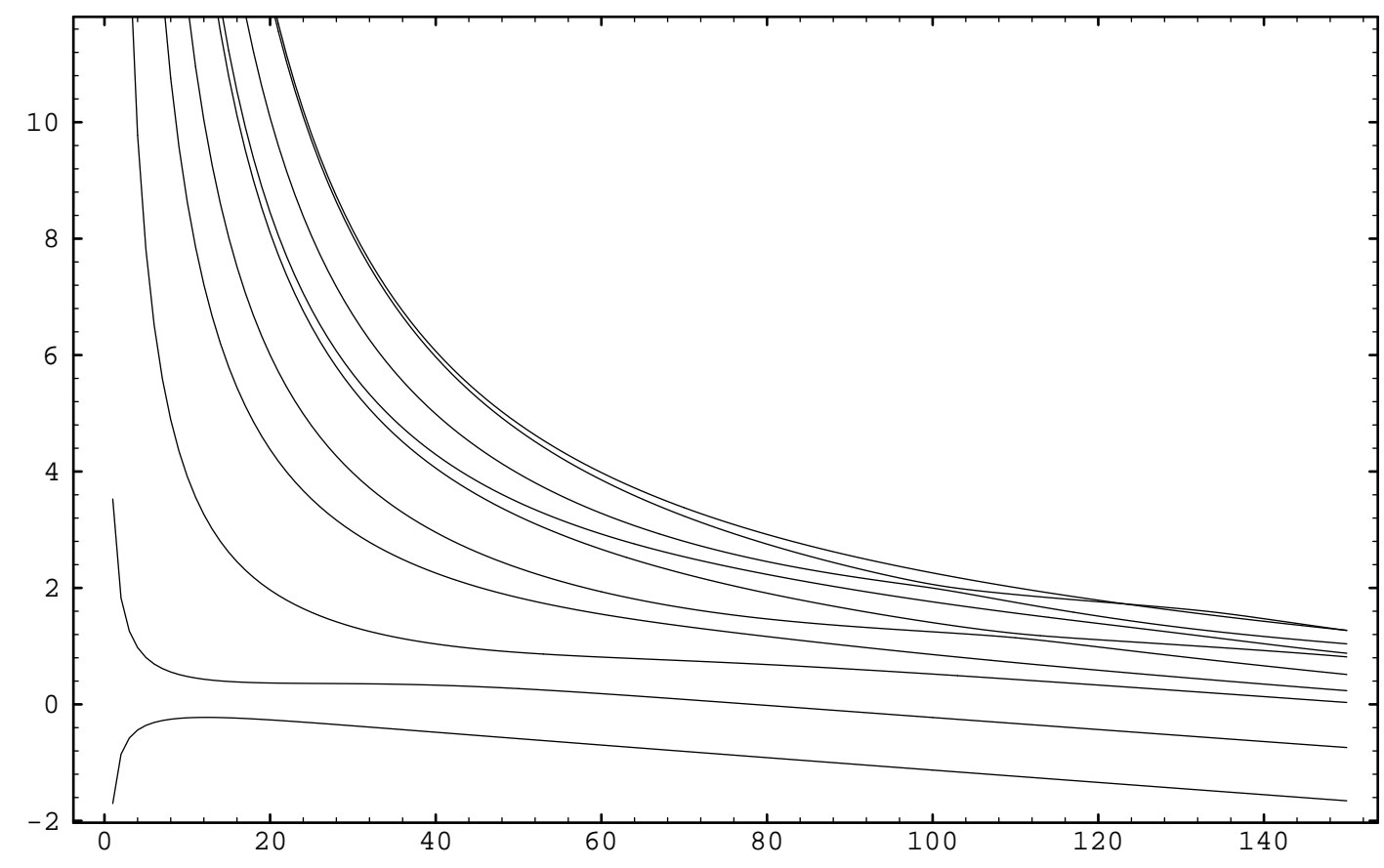

Figure 7.c 


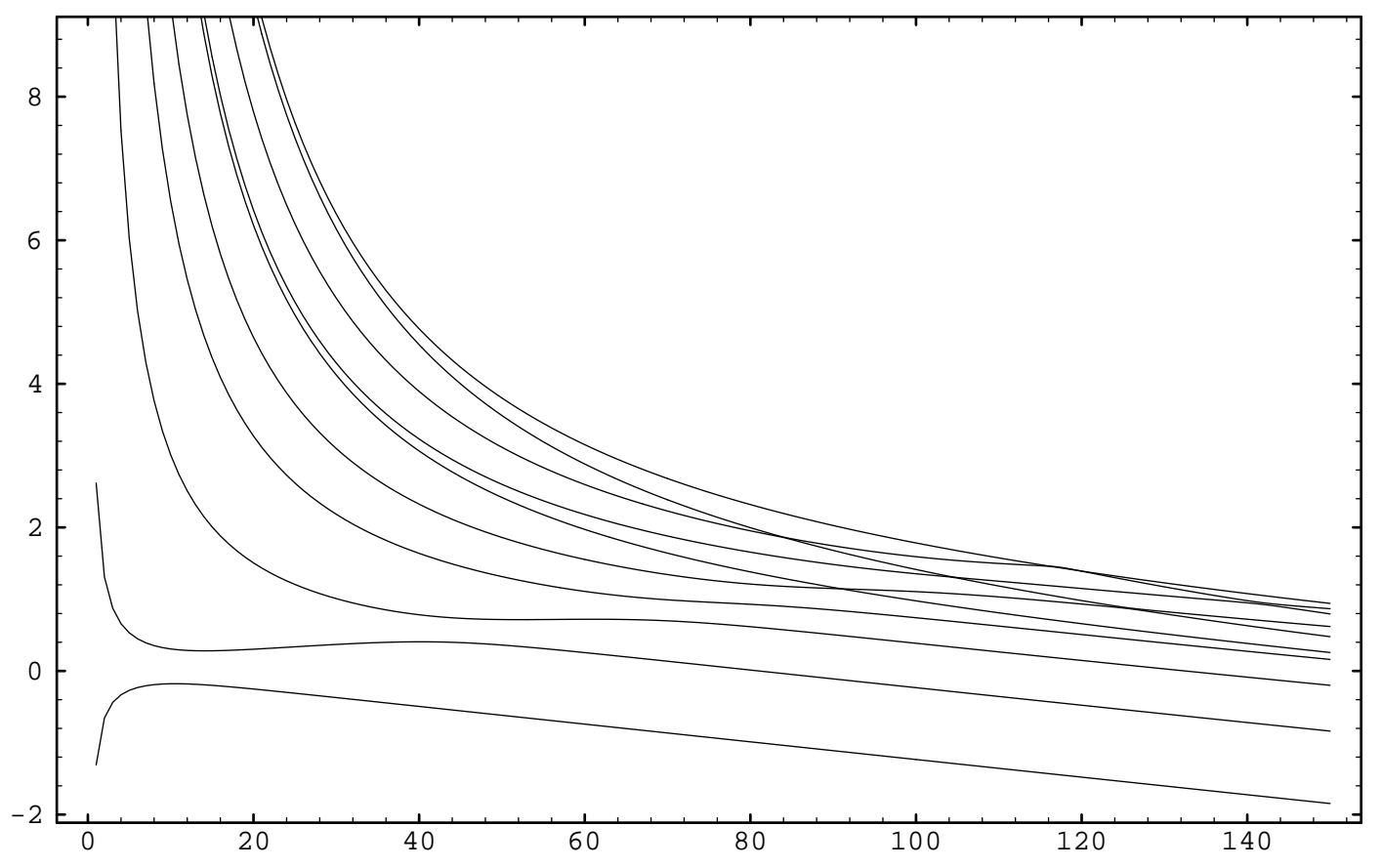

Figure 7.d 


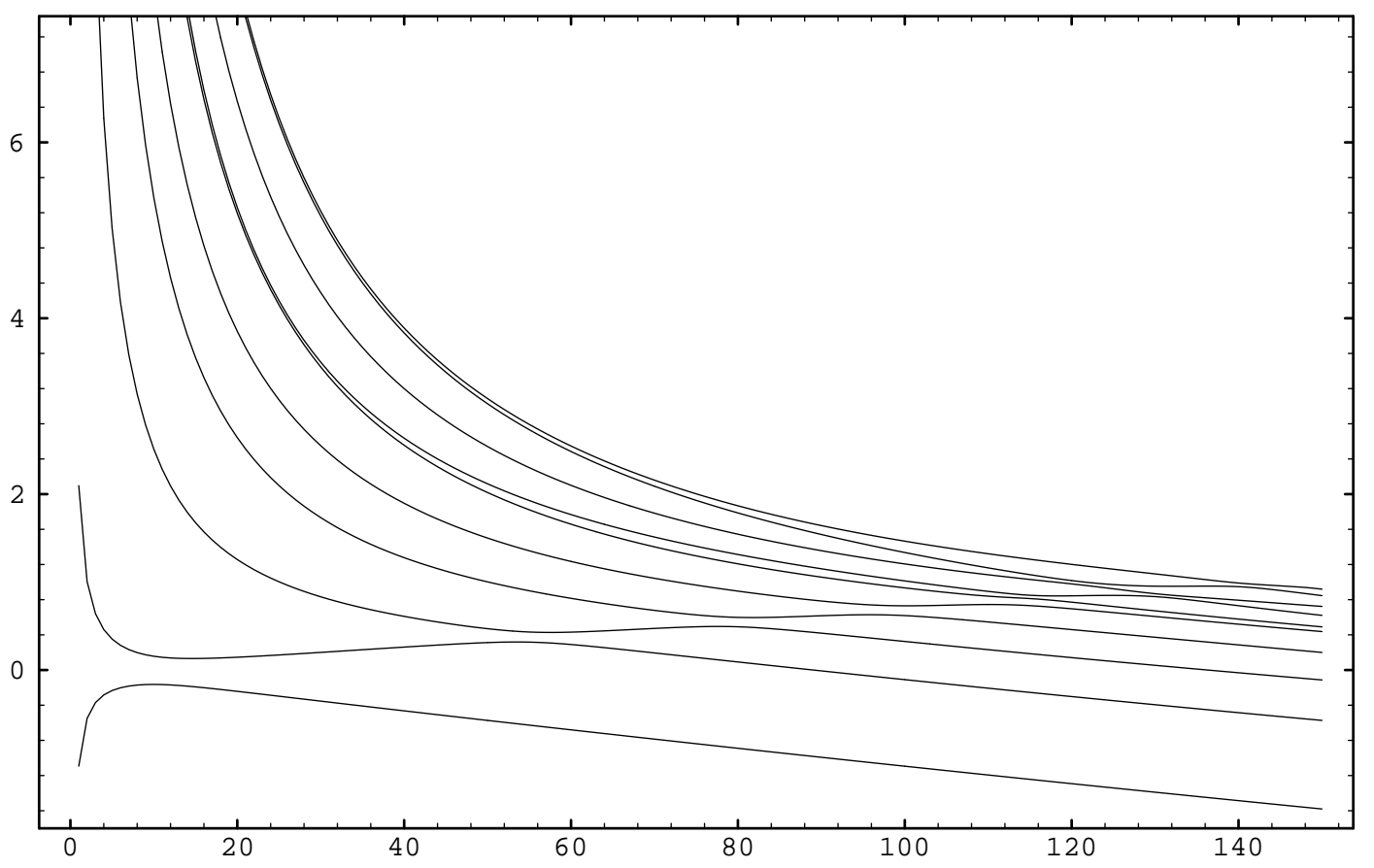

Figure 7.e 


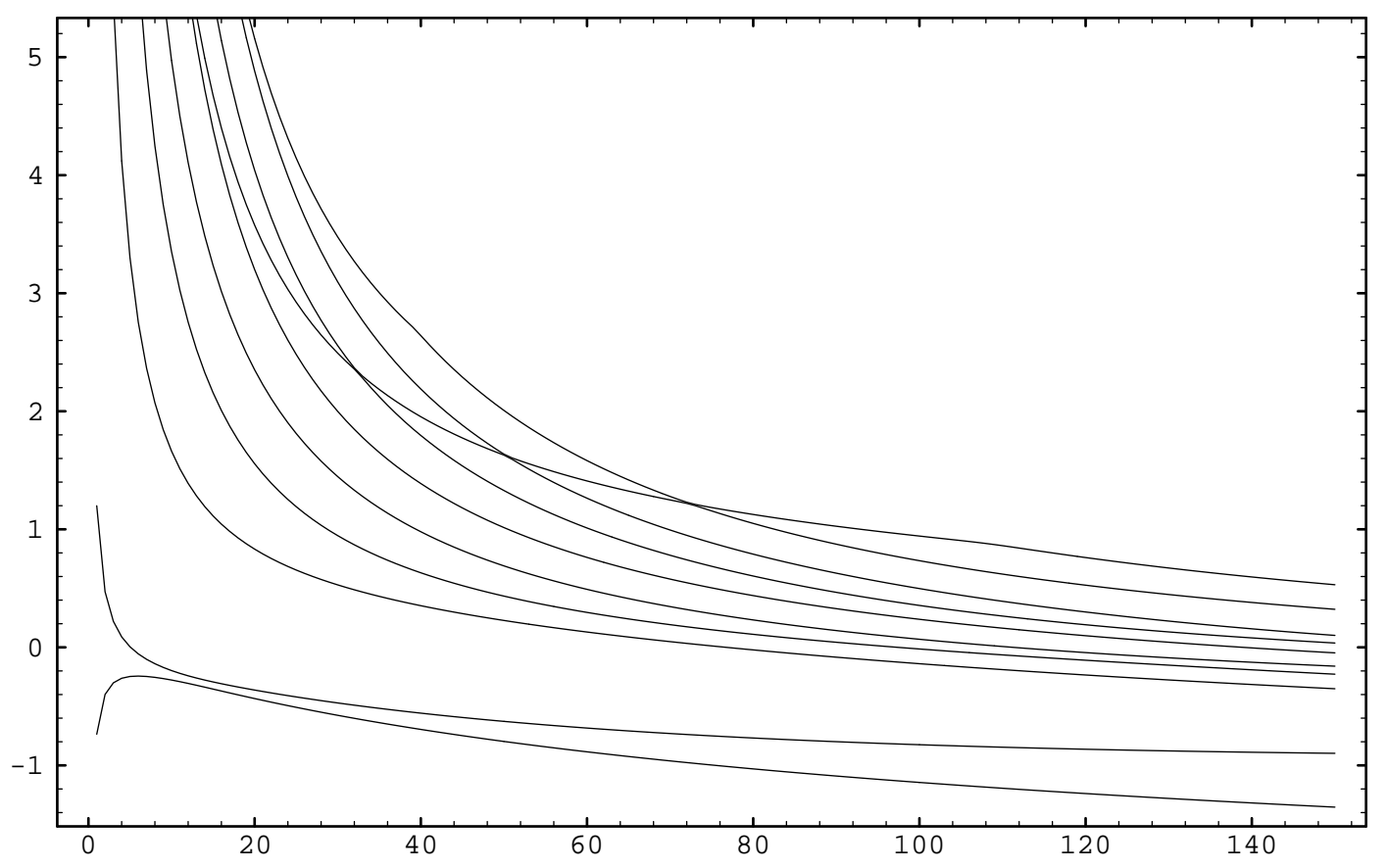

Figure 7.f 


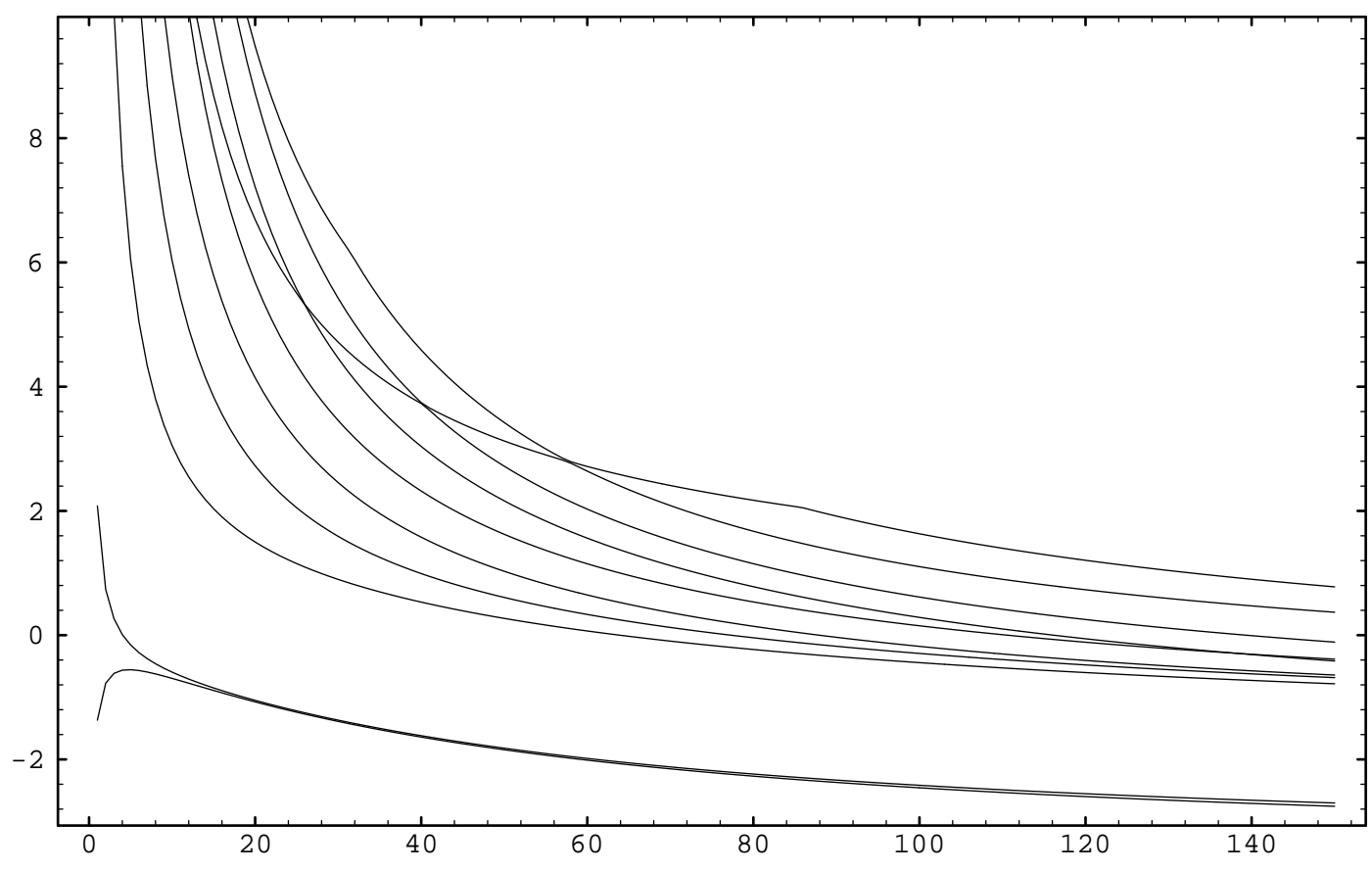

Figure 7.g 


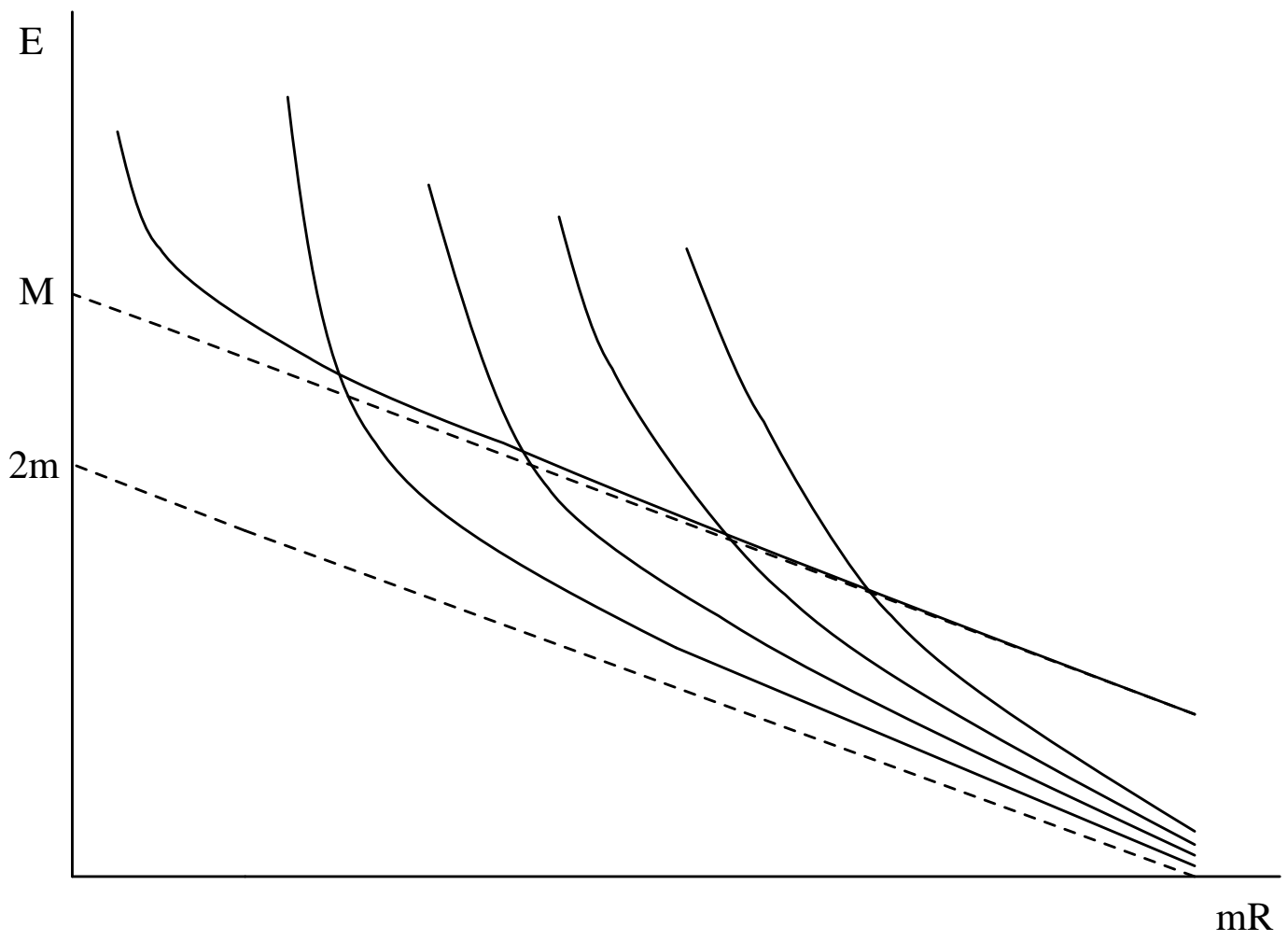

(a)

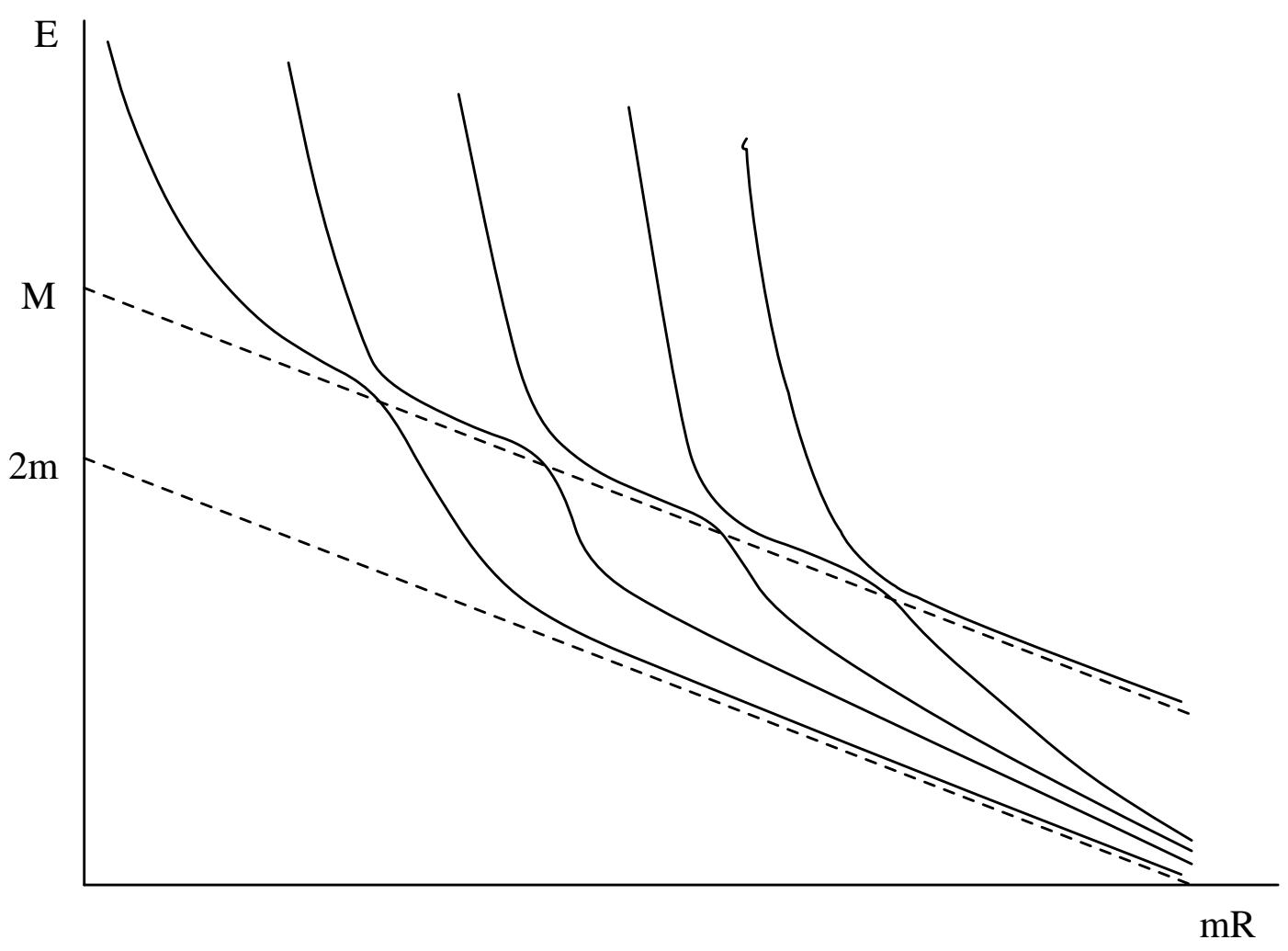

(b)

Figure 8 


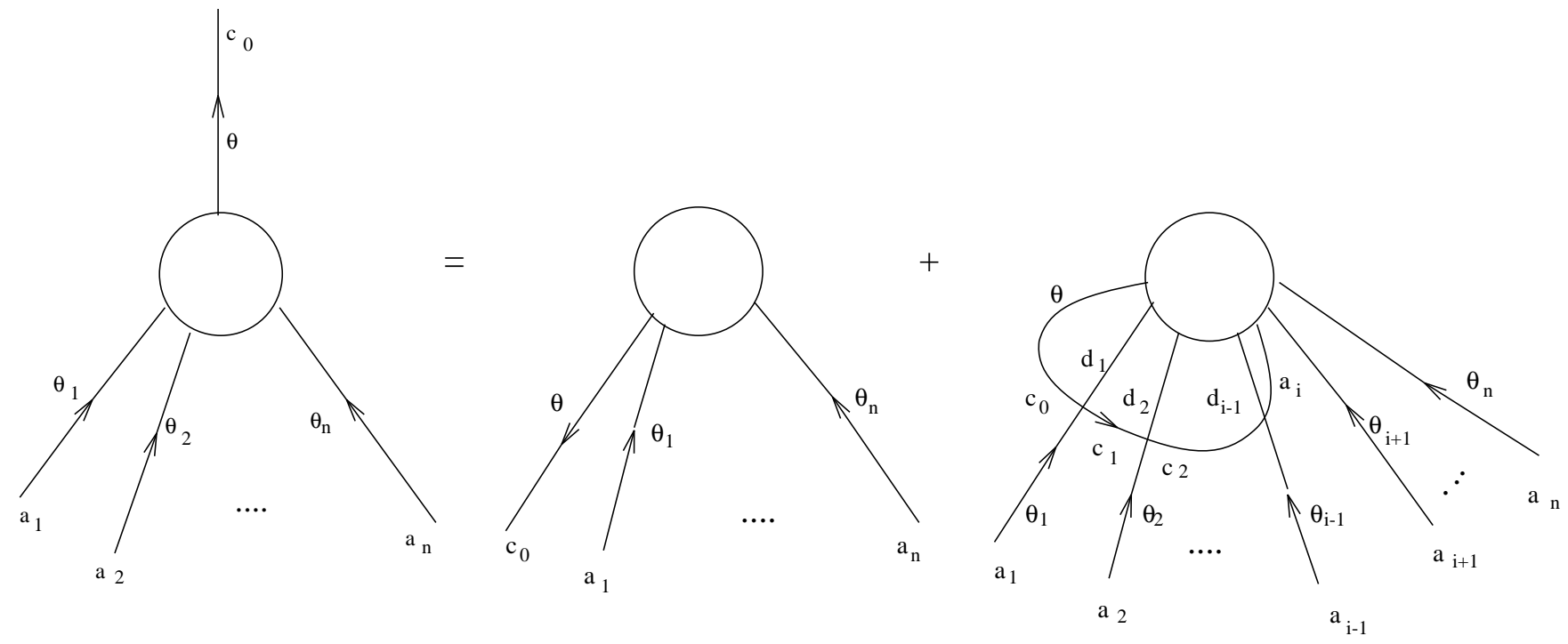

Figure 9 